\title{
X-ray absorption in distant type II QSOs ${ }^{\star}, \star \star$
}

\author{
M. Krumpe ${ }^{1}$, G. Lamer ${ }^{1}$, A. Corral ${ }^{2}$, A. D. Schwope ${ }^{1}$, F. J. Carrera ${ }^{2}$, X. Barcons ${ }^{2}$, \\ M. Page ${ }^{3}$, S. Mateos ${ }^{4}$, J. A. Tedds ${ }^{4}$, and M. G. Watson ${ }^{4}$ \\ 1 Astrophysikalisches Institut Potsdam, An der Sternwarte 16, 14482 Potsdam, Germany \\ e-mail: mkrumpe@aip.de \\ 2 Instituto de Física de Cantabria (CSIC-UC), Avenida de los Castros, 39005 Santander, Spain \\ 3 Mullard Space Science Laboratory, Holmbury St. Mary, Dorking, UK \\ 4 Department of Physics \& Astronomy, University of Leicester, Leicester LE1 7RH, UK
}

Received 6 November 2007 / Accepted 3 March 2008

ABSTRACT

\begin{abstract}
Aims. We present the results of the X-ray spectral analysis of an XMM-Newton-selected type II QSO sample with $z \geq 0.5$ and $0.5-10 \mathrm{keV}$ flux of $0.3-33 \times 10^{-14} \mathrm{erg} / \mathrm{s} / \mathrm{cm}^{2}$. The distribution of absorbing column densities in type II QSOs is investigated and the dependence of absorption on X-ray luminosity and redshift is studied.

Methods. We inspected 51 spectroscopically classified type II QSO candidates from the XMM-Newton Marano field survey, the XMM-Newton-2dF wide angle survey (XWAS), and the AXIS survey to set-up a well-defined sample with secure optical type II identifications. Fourteen type II QSOs were classified and an X-ray spectral analysis performed. Since most of our sources have only $\sim 40 \mathrm{X}$-ray counts (PN-detector), we carefully studied the fit results of the simulated X-ray spectra as a function of fit statistic and binning method. We determined that fitting the spectra with the Cash-statistic and a binning of minimum one count per bin recovers the input values of the simulated X-ray spectra best. Above 100 PN counts, the free fits of the spectrum's slope and absorbing hydrogen column density are reliable.

Results. We find only moderate absorption $\left(N_{\mathrm{H}}=(2-10) \times 10^{22} \mathrm{~cm}^{-2}\right)$ and no obvious trends with redshift and intrinsic X-ray luminosity. In a few cases a Compton-thick absorber cannot be excluded. Two type II objects with no X-ray absorption were discovered. We find no evidence for an intrinsic separation between type II AGN and high X-ray luminosity type II QSO in terms of absorption. The stacked X-ray spectrum of our 14 type II QSOs shows no iron $\mathrm{K} \alpha$ line. In contrast, the stack of the 8 type II AGN reveals a very prominent iron $\mathrm{K} \alpha$ line at an energy of $\sim 6.6 \mathrm{keV}$ and an $E W \sim 2 \mathrm{keV}$.
\end{abstract}

Key words. surveys - X-rays: galaxies - galaxies: active - galaxies: quasar: general

\section{Introduction}

The unification model for active galaxy nuclei (AGN) is based on the assumption that the Seyfert type I/type II dichotomy in AGN is a result of varying orientation relative to the line of sight of similar objects (Antonucci 1993). All AGN consist of a super-massive black hole with an accretion disk. This central engine is surrounded by optically thick, toroidally concentrated dusty material. An observer can either view the central engine directly (type I AGN) or through the optically thick torus (type II AGN). Direct observation of the broad line region reveals a strong UV continuum and broad permitted emission lines while the optical spectra of type II AGN show narrow permitted and forbidden emission lines. Hao et al. (2005) provided a formal separation criterion by finding a bimodal distribution in the $\mathrm{H} \alpha-F W H M$ with a significant dip at $F W H M(\mathrm{H} \alpha)=1200 \mathrm{~km} \mathrm{~s}^{-1}$.

Kleinmann et al. (1988) discovered the first type II quasi stellar object (QSO). QSOs show higher intrinsic luminosity than AGN. In the optical the conventional dividing line between Seyfert galaxies and QSOs is $M_{\mathrm{B}}=-23$. Despite their high intrinsic luminosities, type II QSOs are very hard to identify.

\footnotetext{
* Based on observations obtained with XMM-Newton, an ESA science mission with instruments and contributions directly funded by ESA Member States and NASA.

$\star \star$ Appendix is only available in electronic form at

http://www . aanda.org
}

A significant fraction of the emitted power is absorbed by the optically thick torus. Furthermore, their lack of emission lines in a wide optical wavelength range hampers their identification. About 150 optically selected type II QSOs from the Sloan Digital Sky Survey were studied in Zakamska et al. (2003). However type II QSOs are more efficiently found in followup observations of X-ray surveys (Szokoly et al. 2004; Mainieri et al. 2002; Krumpe et al. 2007; Barcons et al. 2007; Tedds et al. 2008).

Type II QSOs play an important role in understanding the $\mathrm{X}$-ray universe. Since they show significant absorption, type II QSOs/AGN are considered to be a main contributor to the hard $\mathrm{X}$-ray background and their existence in considerable numbers is needed for the synthesis of the X-ray background (Gilli et al. 2007). Possible evolution of the absorption with intrinsic luminosity and/or redshift is a matter of intensive debate and would essentially influence the X-ray background synthesis models.

Many papers discuss the fraction of absorbed AGN as a function of luminosity and/or redshift, and this is a highly controversial subject (Dwelly \& Page 2006; Tozzi et al. 2006; Akylas et al. 2006). However, there are only a few studies (e.g. La Franca et al. 2005) which investigate the evolution of absorbing column densities in type II AGN up to high redshift. Other studies focus on the local universe, where the existence of so-called Compton-thick absorbed objects is well known (Maiolino et al. 1998; Bassani et al. 1999; Risaliti et al. 2000). A Compton-thick 
object is absorbed by column densities of $N_{\mathrm{H}}>1.5 \times 10^{24} \mathrm{~cm}^{-2}$, so that the cross section of Compton scattering overcomes the photo-electric absorption. Hence, the reflected X-ray component is observable.

There is evidence for a large fraction of Compton-thick absorbed quasars at high redshifts (Martinez-Sansigre et al. 2007), but only one candidate has been reported so far at high redshift (Norman et al. 2002), and even in this case the column density is quite uncertain. Most of the X-ray sources from deep Chandra and XMM-Newton surveys are absorbed by column densities $10^{21}<N_{\mathrm{H}} / \mathrm{cm}^{-2}<10^{23}$, with hard X-ray luminosities of $10^{42}<L_{\mathrm{X}_{\mathrm{OBS}}} /(\mathrm{erg} / \mathrm{s})<10^{44}$ (Comastri 2004; Szokoly et al. 2004; Mainieri et al. 2002; Mateos et al. 2005). These values are also found in Compton-thin absorbed $\left(10^{21}<N_{\mathrm{H}} / \mathrm{cm}^{-2}<10^{24}\right)$ Seyfert II galaxies at low redshifts.

The definition of type II QSOs is somewhat arbitrary. Zakamska et al. (2003) define a type II QSO only based on its optical properties. They select AGN with narrow permitted emission lines $\left(F W H M<2000 \mathrm{~km} \mathrm{~s}^{-1}\right)$ and classify objects with [O III] $\lambda 5008$ line luminosity of $L \geq 3 \times 10^{8} L_{\odot}$ as type II QSO. Mainieri et al. (2002) introduce the term "type II QSO region". Although their type I/II classification is based on optical spectra, a type II QSO has to have an intrinsic X-ray luminosity of $L_{\mathrm{X}_{\mathrm{INT}}}>10^{44} \mathrm{erg} / \mathrm{s}(0.5-10 \mathrm{keV})$ and an absorbing hydrogen column density of $N_{\mathrm{H}}>10^{22} \mathrm{~cm}^{-2}$. Throughout the paper we use the definition of a type II QSO to be an object with narrow forbidden and narrow permitted emission lines in the optical spectrum, as well as a de-absorbed intrinsic X-ray luminosity $L_{\mathrm{X}_{\mathrm{INT}}}>10^{44} \mathrm{erg} / \mathrm{s}(0.5-10 \mathrm{keV}$ band). However, the Seyfert type II classification of our sample objects is strictly based on the optical spectra.

The present work focuses on properties of the high X-ray luminosity type II QSOs found in medium deep surveys performed by us. The paper is organised as follows. In Sect. 2 we describe how we selected our type II QSO candidate sample and we study the properties in Sect. 3. The X-ray data and the extraction of the X-ray spectra are described in Sect. 4. Since X-ray spectra with small numbers of counts have to be fitted, we studied different fitting and binning methods in Sect. 5 and then describe the analysis of the X-ray spectra. The results are discussed in Sect. 6. Finally, our conclusions are outlined in Sect. 7.

Unless mentioned otherwise, all errors refer to a $68 \%$ confidence interval. We assume $H_{0}=70 \mathrm{~km} \mathrm{~s}^{-1} \mathrm{Mpc}^{-1}, \Omega_{\mathrm{M}}=0.3$, and $\Omega_{\Lambda}=0.7$.

\section{Definition of the type II QSO sample}

We began with a list of 51 spectroscopically classified type II QSO candidates which are associated with X-ray sources. All optical counterparts have $z \geq 0.5$ and observed X-ray luminosities (not corrected for intrinsic absorption) of $\log \left(L_{\mathrm{X}_{\mathrm{OBS}}}\right)>42.5$. The type II QSO candidates were taken from three different $\mathrm{X}$-ray surveys, the XMM-Newton Marano field survey (Krumpe et al. 2007), the $X M M-2 \mathrm{dF}$ wide angle survey (XWAS, Tedds et al. 2008), and from "An XMM-Newton International Survey" (AXIS, Barcons et al. 2002, 2007; Carrera et al. 2007). To obtain a well-defined sample with secure optical type II identifications and X-ray counterparts we applied a two stage process. First, we visually inspected the optical spectra of all type II QSO candidates from all X-ray surveys and determined the FWHM of the emission lines. The type II QSO candidate classification was only based on the optical spectra. The spectral resolution of the optical setup was $\sim 1050 \mathrm{~km} \mathrm{~s}^{-1}$ for the Marano field survey, $\sim 700 \mathrm{~km} \mathrm{~s}^{-1}$ for XWAS, and $\sim 300-600 \mathrm{~km} \mathrm{~s}^{-1}$ for AXIS. Therefore, an intrinsic line of $1200 \mathrm{~km} \mathrm{~s}^{-1}$ was observed with $\sim 1250-1600 \mathrm{~km} \mathrm{~s}^{-1}$. We defined emission lines with a measured resolution (including instrumental resolution) of $F W H M \leq 1500 \mathrm{~km} \mathrm{~s}^{-1}$ as narrow.

The optical spectra were only used to determine a secure Seyfert type of the objects. They were not used to separate between AGN and QSOs. None of the 51 type II QSO candidates showed obvious broad emission lines in the optical spectra. However, since we aimed for a very strict type II QSO sample, we excluded many objects that showed only the low excitation O II line. The optical spectrum had to comprise several emission lines that allowed us to establish an optically secure classification of the Seyfert type. In addition the signal-to-noise ratio had to be appropriate in order to verify the non-existence of broad permitted emission line. We excluded all doubtful cases.

We introduced two different categories of type II QSO candidates based on the reliability of the type II classification. Objects that have optical spectra with high excitation lines and at least one permitted AGN emission line that is detected but narrow (e.g. Ly- $\alpha, \mathrm{C}$ IV, $\mathrm{H} \beta$ ) are marked with the optical flag "secure" (see Table 1) - signifying a secure optical identification. Their optical classification is very robust.

Objects with less secure type II identification belong to the tentative sample (optical flag "tentative"). The classification as tentative object can be due to the following reasons:

- The optical spectrum shows narrow emission lines, common AGN high excitation lines, but no Mg II emission. Only $[\mathrm{Ne} \mathrm{V}]$ and [O II] are detected in the spectra. The presence of a common AGN high excitation line ([Ne V]) without a broad $\mathrm{Mg}$ II suggests the classification as a type II QSO candidate. However, since no other permitted emission line is accessible, a secure type II classification cannot be established.

- Common AGN high excitation lines are narrow but show indications of underlying broad components (optical spectra in Appendix A). These objects are likely to be transition objects between Seyfert type II/type I.

- The signal-to-noise ratio of the $\mathrm{H} \beta$ line, or its coincidence with the atmospheric band prevents a robust determination of the $F W H M$ and could hide a weak, but broad $\mathrm{H} \beta$ component.

For all sources in the tentative sample we give an individual comment at the end of this section. After inspection of the optical spectra we were left with 14 secure objects and 12 type II objects that belong to the tentative sample.

As a second step to setting up a well-defined type II QSO sample, we verified that the spectroscopically identified counterpart is associated with the X-ray source. For a significant number of objects much deeper imaging data have become available subsequent to the epoch when the spectra were obtained. For example, the XWAS optical counterparts were originally selected from SuperCosmos optical imaging survey data (Hambly et al. 2001). For the majority of the objects we now have additional, deeper imaging data in different bands. These imaging data were obtained with the Wide Field Imager (WFI, $2.2 \mathrm{~m}$ telescope at La Silla) or the Wide Field Camera (WFC, $2.5 \mathrm{~m}$ Isaac Newton telescope on La Palma). We visually investigated the best available imaging data (WFI, WFC, SuperCosmos) for additional optical counterparts and rejected doubtful counterpart identifications. Furthermore, we computed the probability that the optical object with the $R$-band magnitude $m_{\mathrm{R}}$ is associated with the X-ray source. This was based on

$L=\frac{Q \exp \left(-\mathrm{dist}_{\mathrm{OX}}^{2} / 2\right)}{2 \pi \sigma_{\mathrm{X}}^{2} N\left(<m_{\mathrm{R}}\right)}$ 
Table 1. Observed properties of type II QSO candidate sample.

\begin{tabular}{|c|c|c|c|c|c|c|c|c|c|c|}
\hline $\begin{array}{l}\text { (1) } \\
\text { No }\end{array}$ & $\begin{array}{l}\text { (2) } \\
\text { RA }\end{array}$ & $\begin{array}{l}3) \\
\text { Dec }\end{array}$ & $\begin{array}{c}(4) \\
r_{\mathrm{OX}} \\
\\
{[\operatorname{arcsec}]}\end{array}$ & $\begin{array}{l}(5) \\
R\end{array}$ & $\begin{array}{c}(6) \\
z\end{array}$ & $\begin{array}{l}\text { (7) } \\
\text { Opt. } \\
\text { flag }\end{array}$ & $\begin{array}{l}(8) \\
\text { XID } \\
\text { probab. }\end{array}$ & $\begin{array}{l}\text { (9) } \\
\text { CR }\end{array}$ & $\begin{array}{c}(10) \\
\text { Flux } \\
10^{-14} \\
{\left[\mathrm{erg} \mathrm{s}^{-1} \mathrm{~cm}^{-2}\right]}\end{array}$ & $\begin{array}{c}(11) \\
N_{\mathrm{H}_{\mathrm{GAL}}} \\
10^{22} \\
{\left[\mathrm{~cm}^{-2}\right]}\end{array}$ \\
\hline Marano 9A & 31509.9 & -551313 & 1.18 & 22.63 & 1.427 & secure & 0.99 & $21.3 \pm 0.9$ & 3.957 & 0.027 \\
\hline Marano 20A & 31621.5 & -551759 & 0.63 & $23.5^{E}$ & 2.207 & secure & $0.97^{K}$ & $17.1 \pm 1.3$ & 3.014 & 0.027 \\
\hline Marano 32A & 31547.0 & -551755 & 1.44 & 22.96 & 2.727 & tentative & 0.99 & $12.5 \pm 0.9$ & 3.497 & 0.027 \\
\hline Marano 39A & 31339.7 & -550151 & 0.95 & 23.41 & 0.862 & secure & 0.98 & $19.8 \pm 1.5$ & 3.982 & 0.027 \\
\hline Marano 47A & 31538.7 & -551044 & 1.34 & 23.94 & 0.900 & tentative & 0.95 & $4.5 \pm 0.5$ & 1.051 & 0.027 \\
\hline Marano 50A & 31409.9 & -551746 & 1.25 & 23.66 & 0.986 & tentative & 0.96 & $7.6 \pm 0.7$ & 1.667 & 0.027 \\
\hline Marano 51A & 31630.6 & -551503 & 1.83 & 20.95 & 0.58 & tentative & 0.98 & $10.4 \pm 1.0$ & 1.919 & 0.027 \\
\hline Marano 63A & 31516.9 & -550602 & 1.25 & 23.19 & 2.800 & secure & 0.97 & $7.2 \pm 0.7$ & 1.147 & 0.027 \\
\hline Marano 66A & 31500.7 & -550718 & 1.23 & 21.41 & 0.981 & tentative & 0.99 & $4.0 \pm 0.6$ & 0.566 & 0.027 \\
\hline Marano 116A & 31620.7 & -551652 & 1.30 & $21.2^{E}$ & 0.581 & tentative & $0.99^{K}$ & $5.4 \pm 0.9$ & 2.043 & 0.027 \\
\hline Marano 133A & 31426.2 & -552113 & 1.02 & 23.54 & 2.321 & tentative & 0.92 & $2.7 \pm 0.6$ & 0.336 & 0.027 \\
\hline Marano 171A & 31351.4 & -550256 & 2.18 & 22.17 & 0.800 & secure & 0.86 & $5.8 \pm 0.8$ & 1.998 & 0.027 \\
\hline Marano 224B & 31304.8 & -551604 & 1.70 & 21.51 & 0.690 & secure & 0.96 & $8.9 \pm 1.2$ & 3.899 & 0.027 \\
\hline Marano 253A & 31438.0 & -550650 & 2.64 & 21.84 & 0.517 & tentative & 0.65 & $2.6 \pm 0.5$ & 1.341 & 0.027 \\
\hline Marano 463A & 31625.3 & -550839 & 0.55 & $25.0^{E}$ & 2.531 & secure & $0.98^{K}$ & $3.1 \pm 0.8$ & 0.789 & 0.027 \\
\hline Marano 610A & 31551.8 & -551222 & 1.25 & 21.12 & 0.699 & secure & 0.98 & $3.6 \pm 0.6$ & 1.275 & 0.027 \\
\hline X21516_135 & 22626.7 & -043656 & 0.90 & $21.74^{r}$ & 3.278 & secure & 0.91 & $0.9 \pm 0.2$ & 1.574 & 0.025 \\
\hline X00851_154 & 221541.6 & -173754 & 0.64 & 19.00 & 2.976 & secure & 0.96 & $1.7 \pm 0.2$ & 1.210 & 0.023 \\
\hline X01135_126 & 15257.6 & -140840 & 0.91 & $20.87^{U K}$ & 0.543 & secure & $0.97^{W F C}$ & $10.5 \pm 0.5$ & 7.167 & 0.015 \\
\hline X03246_092 & 04346.0 & -202956 & 2.31 & $20.01^{U K}$ & 0.500 & secure & $0.83^{W F C}$ & $3.2 \pm 0.8$ & 4.104 & 0.015 \\
\hline phl5200-001 & 222826.4 & -051820 & 0.63 & $22.0^{r}$ & 0.711 & secure & $0.99^{W F C}$ & $50.0 \pm 1.2$ & 33.03 & 0.053 \\
\hline sds1b-014 & 21843.0 & -050437 & 0.80 & $22.0^{r}$ & 0.962 & tentative & $0.98^{W F C}$ & $6.1 \pm 0.4$ & 6.380 & 0.026 \\
\hline
\end{tabular}

Comments for Table 1:

${ }^{E}$ FORS $R$ pre-imaging magnitude estimate; cross-calibrated with WFI- $R$-band magnitude catalogue; magnitude error estimate \pm 0.5 mag.

${ }^{K}$ Since no $R$-band magnitude catalogue entry existed, the X-ray-to-optical counterpart probability calculation (see Eq. (1)) is based on the $K$-band magnitude catalogue.

${ }^{r}$ SDSS $r$-band magnitude (AB magnitude).

${ }^{U K}$ UK-Schmidt red plate magnitude.

$W F C$ Since no $R$-band magnitude catalogue entry existed, the X-ray-to-optical counterpart probability calculation (see Eq. (1)) is based on the WFC-data.

where dist $_{\mathrm{OX}}$ is the distance between optical counterpart and $\mathrm{X}$-ray source and $\sigma_{\mathrm{X}}$ is the position error of the X-ray source (Sutherland \& Saunders 1992). A detailed description is given in Krumpe et al. (2007). Most of our optical counterparts have probabilities of $>95 \%$ that they are associated with the X-ray source. We rejected objects that have probabilities of less than $65 \%$. All objects in the secure sample have probabilities $\geq 83 \%$.

After the X-ray counterpart verification our sample consists of 22 sources including 13 secure and 9 tentative objects. The separation between AGN and QSOs was made after the determination of the de-absorbed intrinsic X-ray luminosity (see Sect. 5).

Comments on the optical spectra of tentative objects: Marano 32A - all narrow emission lines have underlying broad (mainly blue-shifted) components.

Marano 47A - the signal-to-noise ratio of the optical spectrum does not allow the exclusion of the presence of a weak, broad $\mathrm{H} \beta$ emission line.

Marano 50A - H $\beta$ and [O III] not covered by the spectrum, however, most likely a type II object since no $\mathrm{Mg}$ II but [O II] and $[\mathrm{Ne} \mathrm{V}]$ emission lines.

Marano $51 \mathrm{~A}-\mathrm{H} \beta$ and $[\mathrm{O} \mathrm{III}]$ covered by the spectrum but $\mathrm{H} \beta$ coincides with the edge of the atmospheric A-Band. However, a strong broad $\mathrm{H} \beta$ emission line can be ruled out and [O II], $[\mathrm{Ne} \mathrm{V}]$ emission lines are visible.

Marano 66A - SNR of the optical spectra does not exclude the presence of a weak, broad $\mathrm{H} \beta$ emission line ([O II] and [Ne V] emission).

Marano 116A - type II object/X-ray bright optical normal galaxy (XBONG) - galaxy spectrum with prominent [O II] emission line, no $\mathrm{Mg}$ II, very weak [O III] emission lines, $\mathrm{H} \beta$ coincides with the edge of the A-Band.

Marano 133A - some narrow emission lines have underlying broad, blue-shifted components.

Marano 253A - type II object/XBONG - no Mg II and $\mathrm{H} \beta$ emission, weak [O III] emission, the second [O III] line falls into the atmospheric A-band.

sds1b-014 - high SNR spectrum, $\mathrm{H} \beta$ and [O III] not covered by the spectrum, however most likely a type II object since no Mg II but [O II] and [Ne V] emission lines.

\section{Properties of the type II QSO candidate sample}

In Table 1 we summarise the observed properties of the objects. We list the name of the spectroscopically identified counterpart, optical coordinates, distance between spectroscopically identified counterpart and X-ray source, the WFI- $R$-band magnitude (unless otherwise mentioned), redshift, optical flag, X-ray counterpart probability, count rate, $0.5-10 \mathrm{keV}$ flux, and the Galactic absorption along the line of sight.

The objects cover a redshift range of $0.5 \leq z \leq 3.278$. Since the lack of emission lines in a wide optical wavelength range hampers the identification of type II objects, we only have one type II QSO candidate in the redshift interval of $z=1-2$. Figure 1 shows the $R$-band magnitude histogram of the selected objects. As a simplification we consider the $r$-band (SDSS) magnitude to be equal to the $R$-band magnitude, although we are aware that shifts of 0.5 mag between $R$ and $r$ (SDSS) may occur. 


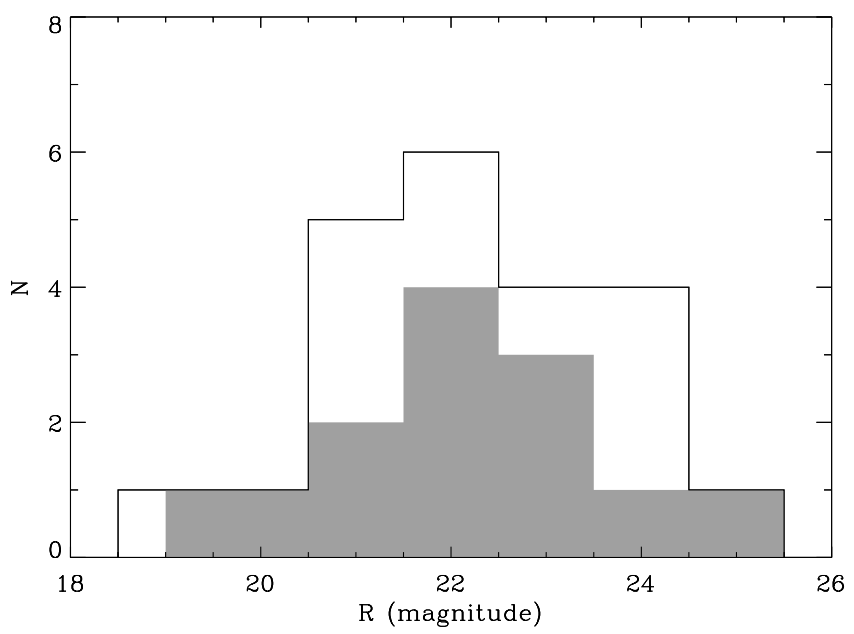

Fig. 1. $R$-band magnitude histogram of all 22 type II QSO candidates (black solid line) and of the 13 secure type II QSO candidates (grey filled histogram).

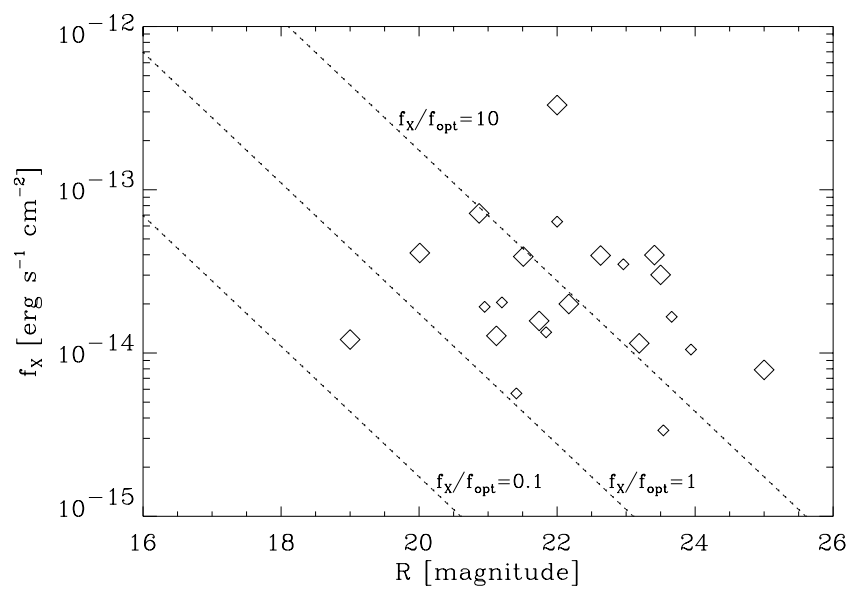

Fig. 2. Observed $0.5-10 \mathrm{keV}$ X-ray flux vs. $R$-band magnitude. Large symbols represent secure type II QSO candidates while small symbols illustrate tentative type II QSO candidates. Dashed lines indicate $f_{\mathrm{X}} / f_{\mathrm{OPT}}$ values of $0.1,1$, and 10 .

The majority of our sources have $21<R<24$. The $0.5-10 \mathrm{keV}$ flux in the sample ranges from $0.3-33 \times 10^{-14} \mathrm{erg} / \mathrm{s} / \mathrm{cm}^{2}$.

To calculate $f_{\mathrm{X}} / f_{\mathrm{OPT}}$ values we derived the optical fluxes in a band centred at $7000 \AA$ with a width of $1000 \AA$ using the equation $f_{\text {opt }}=10^{-0.4 R-5.759}$ (Zombeck 1990). X-ray fluxes were calculated for the $0.5-10 \mathrm{keV}$ energy range. The $R$-band magnitude vs. X-ray flux plane (Fig. 2) clearly shows AGN activity in all selected sources, since they have X-ray-to-optical flux ratios of $f_{\mathrm{X}} / f_{\text {OPT }}>0.25$. In accordance with Mainieri et al. (2002) we consider objects with $f_{\mathrm{X}} / f_{\mathrm{OPT}}>0.1$ as AGN. Almost half of the selected type II QSO candidates have noticeable high X-ray-tooptical flux ratios $\left(f_{\mathrm{X}} / f_{\mathrm{OPT}}>10\right)$. Szokoly et al. (2004) mentioned that type II AGN/QSOs cluster at higher X-ray-to-optical flux ratios than type I AGN. The majority of the spectroscopically classified type II AGN/QSOs in the Lockman Hole also show high $f_{\mathrm{X}} / f_{\mathrm{OPT}}$ values (Mainieri et al. 2002). The observed 0.5-10 keV X-ray luminosities (not corrected for intrinsic absorption) of our objects range from $L_{\mathrm{X}_{\mathrm{OBS}}} \sim 10^{43-45} \mathrm{erg} / \mathrm{s}$.

\section{XMM-Newton observations and extraction of X-ray spectra}

All data were processed with the SAS version 7.0 (Science Analysis Software, Gabriel et al. 2004) package and corresponding calibration files. The epchain and emchain tasks were used for generating linearised event lists from the raw PN and MOS data. For all sources the effects of photon pile-up was negligible.

The XMM-Newton data reduction for the Marano field survey is described in detail in Krumpe et al. (2007). For the XWAS and AXIS sources we downloaded all available X-ray data from the XMM-Newton archive ${ }^{1}$ up to and including July 2007. Periods of high background were excluded from the analysis of all relevant data sets in the standard way.

Circular or box-shaped source and background regions were manually determined for all contributing observations. Sources at large off-axis angles in the contributing observations were not considered as follows. The largest offaxis angles were 720 arcsec for the PN and 820 arcsec for the MOS detectors, respectively. The lower PN area was a result of the enhanced background contamination near the edges of the PN detector. Additional X-ray sources in the background regions were masked out. The auxiliary response file (arf) was computed for each source and observation individually. For the Marano field sources, we used appropriately "canned" response matrices from the XMM-Newton calibration homepage ${ }^{2}$ (XMM-revolution 110 and pattern $0-12$ ). The PN $y$-coordinate of an X-ray source was determined to link the relevant $\mathrm{PN}$ response matrix file ( $\mathrm{rmf}$ for single and double events, version 6.8) to the X-ray spectrum of the source. For the XWAS and AXIS sources the response matrix files were computed on a case-by-case basis. Where multiple X-ray spectra of a given source were added, the mean rmf was computed as a weighted mean. For details of the procedure see Page et al. (2003). The two MOS spectra were always added to form a single MOS spectrum.

The X-ray source of the secure type II object phl5200-001 is surrounded by diffuse X-ray emission. For the reduction of the $\mathrm{X}$-ray spectrum we used a smaller extraction radius for the point source and the diffuse X-ray emission as the background region.

\section{X-ray spectral analysis}

The X-ray spectral analysis was performed with XSPEC (Arnaud 1996) version 12.3.0. Although we have a few objects with several hundreds of net PN source counts in the $0.2-8 \mathrm{keV}$ range, the distribution peaks at $\sim 40$ net PN source counts. An appropriate X-ray spectral analysis for the low count regime has to be found. Although we only refer to the net PN counts in our simulations and in Table 4, the fit uses both the PN and MOS data. Hence, the total number of counts used by the fit is typically twice that given in Table 4.

\subsection{Defining the appropriate fit statistic and binning method}

A problem notorious to X-ray astronomy is proper fitting of spectra with relatively few counts. Tozzi et al. (2006) approached this problem for the X-ray sources in the Chandra Deep Field South by running simulations for two different fitting procedures: Cash-statistics (unbinned) and classic $\chi^{2}$-statistics with a binning of 10 counts per bin (min 10). They concluded that the

\footnotetext{
1 http://xmm.esac.esa.int/xsa

2 http://xmm.esac.esa.int/external/xmm_sw_cal/calib
} 
Table 2. Set of input parameters for the simulated X-ray spectra.

\begin{aligned} & \hline \hline Parameter value \\ & \hline $0.2-8 \mathrm{keV}$ net counts $10,40,100,130,200 \\ &$ Redshift $1,2,3 \\ &$ Column density $\left[\mathrm{cm}^{-2}\right] 0,10^{21}, 10^{22}, 10^{23}, 10^{24} \\ &$ Photon index $\Gamma 2.0 \\ &$\hline\end{aligned}

Table 3. Fit statistics and binning methods for the simulated X-ray spectra.

\begin{aligned} & \hline \hline Fit statistic binning methods \\ & \hline Cash-statistic unbinned, min 1, min 2, min 3, \\ & min 5, min 10, min 15, channel 60, \\ & channel 120, channel 180, channel 240 \\ &$\chi^{2}$-statistic min 10, min 15 \\ & Photon index $\Gamma$ free fit, 2.0 \\ & \hline\end{aligned}

The data were grouped from a minimum PHA channel (corresponding to $0.2 \mathrm{keV}$ ) to the maximum PHA channel (corresponding to $8 \mathrm{keV}$ ), with at least $n$ counts per bin (abbreviated as $\min n$ ) or with a fixed number $n$ of channels (channel $n$ ).

unbinned Cash-statistic fits recovered the input values better for $\mathrm{X}$-ray spectra with less than 50 counts.

We carried out a much more extensive study of the fit results as a function of fit statistic and binning method. This investigation can be used to study the error distribution of the intrinsic column density $\left(N_{\mathrm{H}}\right)$ and to determine how many PN net counts are required to perform free fits of $N_{\mathrm{H}}$ and photon index $\Gamma$ with acceptable errors in both parameters.

We assumed emission from the AGN to be described as a power law with photon index $\Gamma$, modulated by Galactic foreground absorption and further intrinsic cold absorption at the redshift of the AGN. Following Mainieri et al. (2002) and Mateos et al. (2005), we simulated X-ray spectra with an input value of $\Gamma=2$. All simulated $X$-ray spectra included Galactic absorption of $N_{\mathrm{H}_{\mathrm{GAL}}}=2 \times 10^{20} \mathrm{~cm}^{-2}$ and considered all possible parameter combinations of Table 2.

The X-ray spectra were normalised to reach the desired 0.2-8 keV PN net counts with a deviation of up to $5 \%$. We added Poisson noise to the X-ray spectra. We used a typical representative background file (Marano 9A). As for the real data we added MOS1 and MOS2 spectra to form a single MOS spectrum. The same source was simulated 1000 times for each set of specific parameters.

The simulated X-ray spectra were grouped by using different binning methods (Table 3 ). Cash and $\chi^{2}$-statistic were applied to recover the $N_{\mathrm{H}}$ input value. The redshift and the Galactic absorption were set to the input value. The nominal initial guess for the intrinsic absorption was $N_{\mathrm{H}}=0 \mathrm{~cm}^{-2}$, but other initial guesses up to $N_{\mathrm{H}}=10^{24} \mathrm{~cm}^{-2}$ were tested as well.

We studied the recovered $N_{\mathrm{H}}$ distribution with different fit statistics and binning methods by analysing the number of mismatches, the peak, the shape and the significance of $N_{\mathrm{H}}$ detection. The best fit statistic and binning method was selected by these criteria in the given order. In summary, the most important results of our simulations are as follows.

- With only very few exceptions, the Cash-statistic with a binning of one count per bin (min 1) recovered the input values best.

- Even at a level of 40 net PN counts, the Cash-statistic with a binning of minimum one count per bin correctly recovers more than $90 \%$ of the objects with an intrinsic

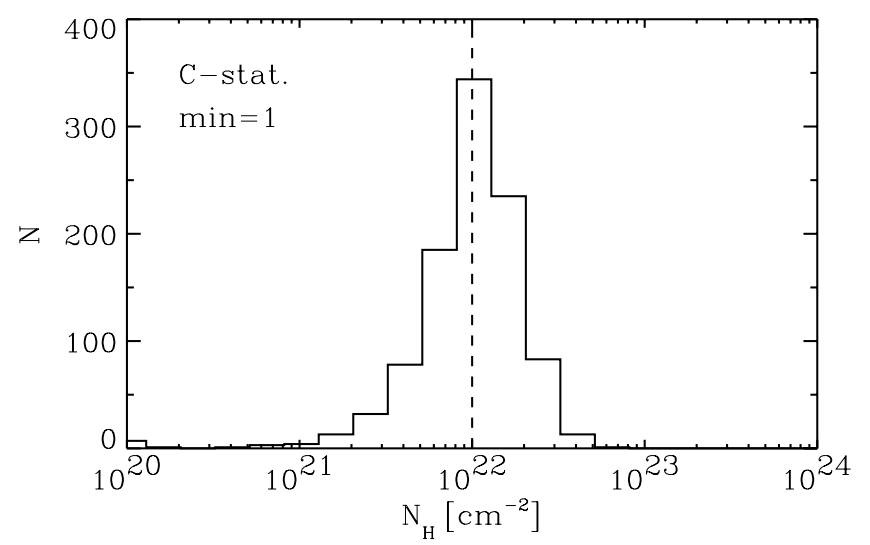

Fig. 3. Recovered $N_{\mathrm{H}}$ distribution for an X-ray source with 40 net PN counts at $z=2$. The input $N_{\mathrm{H}}=10^{22} \mathrm{~cm}^{-2}$ is indicated by the dashed line. Fit method used: fixed $\Gamma=2$, Cash-statistic, binning: $\min 1$; 1000 simulations.

absorption of $N_{\mathrm{H}} \sim 10^{22}-10^{24} \mathrm{~cm}^{-2}$ up to $z=3.5$. Figure 3 shows the retrieved $N_{\mathrm{H}}$ distribution for an input value of $N_{\mathrm{H}}=10^{22} \mathrm{~cm}^{-2}$ at $z=2$. The variance of the recovered $N_{\mathrm{H}}$ distribution increases with redshift. However, individual peaks clearly separate in the studied redshift range, when the intrinsic $N_{\mathrm{H}}$ values differ by a factor of approximately 10 in hydrogen column density. The recovered $N_{\mathrm{H}}$ distribution for an intrinsically unabsorbed source at $z=1$ is shown in Fig. 4. Between 50-60\% of all objects are correctly recovered independent of redshift. Although not all fits converge at $N_{\mathrm{H}}=0 \mathrm{~cm}^{-2}$, none of the resulting $N_{\mathrm{H}}$ values is significantly different from zero. The peak of the misclassified absorbed objects shifts from $N_{\mathrm{H}} \sim 10^{21} \mathrm{~cm}^{-2}$ for $z=1$ to $N_{\mathrm{H}} \sim 7 \times 10^{21} \mathrm{~cm}^{-2}$ for $z=3$.

- For 10 net PN counts the fit retrieves a very broad $N_{\mathrm{H}}$ distribution for objects with an intrinsic absorption of $N_{\mathrm{H}} \sim 10^{22}-10^{24} \mathrm{~cm}^{-2}$. Independent of redshift the fit recovers roughly $30 \%$ of the intrinsically absorbed X-ray sources as unabsorbed sources $\left(N_{\mathrm{H}}=0 \mathrm{~cm}^{-2}\right)$. However, the fit does not significantly overpredict the $N_{\mathrm{H}}$ values. Although no absorption is recovered with a significance above $2 \sigma$ due to the large errors, the fitted $N_{\mathrm{H}}$ values can be used as an estimate for the intrinsic $N_{\mathrm{H}}$ values. An unabsorbed X-ray source is correctly recovered by the fit in $50-60 \%$ of all cases. In $90 \%$ of the cases the fitted $N_{\mathrm{H}}$ values for an intrinsically unabsorbed X-ray source do not exceed $N_{\mathrm{H}} \sim 10^{22} \mathrm{~cm}^{-2}$ for $z=1$ and $N_{\mathrm{H}} \sim 3 \times 10^{22} \mathrm{~cm}^{-2}$ for $z=2$.

- The unbinned Cash-statistic as used in Tozzi et al. (2006) shows the narrowest distribution in $N_{\mathrm{H}}$ for most of the parameter combinations, but the peak of the recovered distribution was found to be strongly dependent on the initial guess of $N_{\mathrm{H}}$ and weakly favours $N_{\mathrm{H}}=10^{22} \mathrm{~cm}^{-2}$ independent of input parameter for net PN counts less than 100 .

- For a binning of min 10 or min 15 we find no difference between the Cash and the $\chi^{2}$-statistic even at a level of 40 net PN counts. Only the significance of the absorption is marginally higher for the Cash-statistic. There is also no difference between a binning of min 10 and min 15 .

- Free fits in $N_{\mathrm{H}}$ and $\Gamma$ are acceptable above $100 \mathrm{PN}$ net counts. The recovered $N_{\mathrm{H}}$-values show a narrow distribution at the input $N_{\mathrm{H}}$ value $(F W H M \sim 0.4 \mathrm{dex})$. For all combinations of fitting and binning methods we retrieve the peak of 


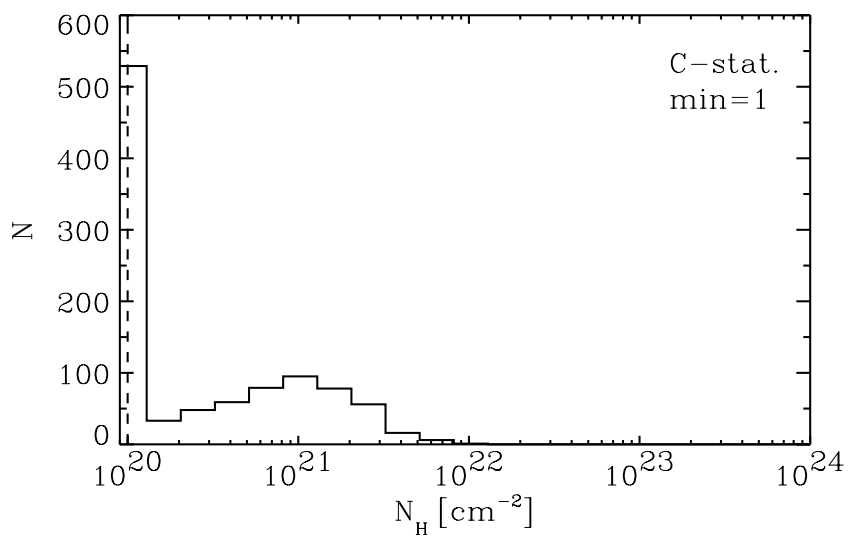

Fig. 4. Recovered $N_{\mathrm{H}}$ distribution of an unabsorbed X-ray source with 40 net PN counts at $z=1$. The input $N_{\mathrm{H}}=0 \mathrm{~cm}^{-2}$ is indicated by the dashed line. $N_{\mathrm{H}}=0 \mathrm{~cm}^{-2}$ is represented by the lowest bin. Fit method used: fixed $\Gamma=2$, Cash-statistic, binning: $\min 1$; 1000 simulations.

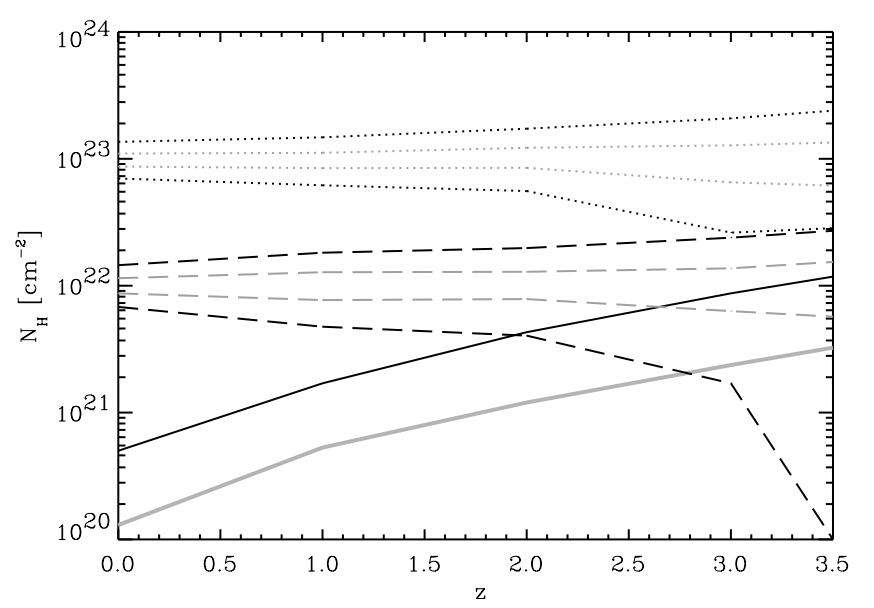

Fig. 5. Diagnostic $N_{\mathrm{H}}$ vs. redshift plot. The plotted contours contain $68 \%$ (grey) and $90 \%$ (black) of the simulated X-ray sources with 40 net PN counts in the $0.2-8 \mathrm{keV}$ band (NOTE: The fits used more than twice as many counts since both the PN and MOS data are used). The solid lines are the limits for intrinsically unabsorbed sources. The limits for an intrinsic absorption of $N_{\mathrm{H}}=10^{22} \mathrm{~cm}^{-2}$ are shown by dashed lines while dotted lines represent $N_{\mathrm{H}}=10^{23} \mathrm{~cm}^{-2}$.

$\Gamma \simeq 2$. For a binning of minimum one count per bin $(\min 1)$ the recovered $\Gamma$ distribution clearly peaks at $\Gamma=2$ with $F W H M \sim 0.8\left(F W H M_{130 \text { counts }} \sim 0.4, F W H M_{200 \text { counts }} \sim 0.2\right)$.

Simulations as shown in Figs. 3 and 4 are used to determine the $N_{\mathrm{H}}$ range that belongs to $68 \%$ and $90 \%$ of all simulations. In Fig. 5 we show these distributions for simulations at different redshifts $(z=0,1,2,3,3.5)$ and column densities $\left(N_{\mathrm{H}}=0,10^{22}, 10^{23} \mathrm{~cm}^{-2}\right)$. The simulations contain 40 net PN counts and so are representative of many of our sources. The Cash-statistic with a binning of at least one count per bin is an appropriate method to determine the column density over a wide range of redshifts. It does not significantly overestimate the input $N_{\mathrm{H}}$ values. The fit recovers more than $90 \%$ of the unabsorbed sources up to $z=3$ with $N_{\mathrm{H}}$ values below $N_{\mathrm{H}}=10^{22} \mathrm{~cm}^{-2}$.

\subsection{Modelling the $X$-ray spectra}

Following our analysis in Sect. 5.1 we grouped the extracted $\mathrm{X}$-ray spectra in bins of at least one count per bin $(0.2-8 \mathrm{keV})$ and used the Cash-statistic to determine $N_{\mathrm{H}}$. The initial guess was set to $N_{\mathrm{H}}=0$. We corrected for Galactic absorption and performed free fits in $N_{\mathrm{H}}$ and $\Gamma$ for spectra with more than 100 net PN counts. For X-ray spectra with less than 100 net PN counts we fixed $\Gamma=2$ and only fit $N_{\mathrm{H}}$. In these cases we give two errors for the value of $N_{\mathrm{H}}$ in Table 4 . The first is the $1 \sigma$ error of $N_{\mathrm{H}}$ based on the fit with fixed $\Gamma=2$, while the second takes into account the systematic shift for different photon indices (deviation in $N_{\mathrm{H}}$ for a fixed $\Gamma=1.7$ and $\Gamma=2.3$ ). The observed (not corrected for intrinsic absorption) and the intrinsic $0.5-10 \mathrm{keV}$ X-ray luminosities were computed by either a free fit in $N_{\mathrm{H}}$ and $\Gamma$ for the bright sources (net PN counts $>100$ ) or a fixed $\Gamma=2$ fit for the faint sources.

Due to contamination by soft detector background we only used the $0.3-8 \mathrm{keV}$ band for the X-ray spectral analysis of X-ray source X03246_092 (24 net PN counts in $0.3-8 \mathrm{keV}$ band). The soft energies in the X-ray spectra of Marano 51A and Marano $610 \mathrm{~A}$ could not be well fitted by an single absorbed power law. In these cases a fit of an absorbed power law plus a soft excess component reproduce the X-ray data better. Therefore, we used a two component fit, an absorbed power law and an unabsorbed power law. No evidence for soft excess was found in the X-ray spectra of the brightest objects. The best fit models of the X-ray data are shown in Appendix A.

Table 4 shows the spectral parameters. In the columns we list the name of the spectroscopically identified counterpart (1), the XMMU source name (2) of the XMM-Newton X-ray source, the observed (3) and intrinsic (4) X-ray luminosity, the PN count number (5) in the $0.2-8 \mathrm{keV}$ band, the absorbing hydrogen column density (6), the photon index (7), and the quality for the used fit (8) when the X-ray data are modelled by a power law plus intrinsic absorption model, and finally, the photon index (9) and the quality for a purely reflection-dominated model (10). If no error is given for the photon index, this parameter was fixed for the fitting.

\section{Discussion}

In Fig. 6 we show the fitted intrinsic $N_{\mathrm{H}}$ distribution of the sources. A column density peak at $N_{\mathrm{H}}=4 \times 10^{22} \mathrm{~cm}^{-2}$ is found. We find moderate absorption in the majority of our objects. The significance of the absorption exceeds $2 \sigma$ in most of the cases (see the confidence contours in Appendix A). Two type II objects are consistent with being unabsorbed X-ray sources, one of which is from the secure type II sample.

The determination of the intrinsic $N_{\mathrm{H}}$ also allows us to compute the de-absorbed intrinsic X-ray luminosity $L_{\mathrm{X}_{\mathrm{INT}}}$ of our sources. As mentioned in Sect. 1, we define a type II object by the detection of narrow emission lines in the optical spectrum. Figure 7 shows the intrinsic column density vs. deabsorbed intrinsic X-ray luminosity plane. The dividing line of $L_{\mathrm{X}_{\mathrm{INT}}}=10^{44} \mathrm{erg} / \mathrm{s}$ is used to distinguish between type II AGN and QSOs. Ten type II objects from the secure sample and four tentative objects are identified as QSOs. We detected one unabsorbed type II QSO but the object belongs to the tentative sample. No obvious trend of absorption in type II QSOs with intrinsic X-ray luminosity is found.

Most of the type II QSOs fall into the same region of the $N_{\mathrm{H}}-L_{\mathrm{X}_{\mathrm{INT}}}$ diagram where previous studies have also found type II QSOs (Mainieri et al. 2002; Szokoly et al. 2004; La Franca et al. 2005; Ptak et al. 2006). The additional criterion $\left(N_{\mathrm{H}}>10^{22} \mathrm{~cm}^{-2}\right.$, Mainieri et al. 2002) makes only a small difference to our sample selection (two more secure objects).

The $N_{\mathrm{H}}$-redshift plane for type II QSOs and type II AGN is shown in Fig. 8. We found no obvious differences between the 
Table 4. Computed properties of type II QSO candidate sample.

\begin{tabular}{|c|c|c|c|c|c|c|c|c|c|}
\hline $\begin{array}{l}\text { (1) } \\
\text { No }\end{array}$ & $\begin{array}{c}(2) \\
\text { XMMU J }\end{array}$ & $\begin{array}{c}(3) \\
\log \left(L_{\mathrm{X}_{\mathrm{OBS}}}\right) \\
0.5-10 \mathrm{keV}\end{array}$ & $\begin{array}{c}(4) \\
\log \left(L_{\mathrm{X}_{\mathrm{INT}}}\right) \\
0.5-10 \mathrm{keV}\end{array}$ & $\begin{array}{c}(5) \\
N_{\text {Counts }} \\
\text { PN }\end{array}$ & $\begin{array}{c}(6) \\
N_{\mathrm{H}} \\
10^{22} \\
{\left[\mathrm{~cm}^{-2}\right]}\end{array}$ & $\begin{array}{c}(7) \\
\Gamma \\
\text { intr. } \\
\text { absor. }\end{array}$ & $\begin{array}{c}(8) \\
\text { C-stat/d.o.f. } \\
\text { intr. } \\
\text { absor. }\end{array}$ & $\begin{array}{c}(9) \\
\Gamma \\
\text { pexrav }\end{array}$ & $\begin{array}{c}(10) \\
\text { C-stat/d.o.f. } \\
\text { pexrav }\end{array}$ \\
\hline Marano 9A & $031510.1-551313$ & 44.50 & 44.55 & 270 & $0.3 \pm 0.1$ & $1.7 \pm 0.1$ & $426 / 503$ & $2.2 \pm 0.3$ & $473 / 503$ \\
\hline Marano 20A & 031621.6-551759 & 44.82 & 44.99 & 64 & $1.7 \pm 0.4 \pm 0.7$ & 2.0 & $183 / 227$ & 2.0 & $216 / 227$ \\
\hline Marano 32A & $031547.2-551755$ & 44.88 & 45.22 & 129 & $9.8 \pm 2.4$ & $1.8 \pm 0.2$ & $285 / 291$ & $2.3 \pm 0.6$ & $295 / 291$ \\
\hline Marano 39A & 031339.7-550151 & 44.04 & 44.22 & 36 & $0.8 \pm 0.2 \pm 0.4$ & 2.0 & $111 / 117$ & 2.0 & $136 / 117$ \\
\hline Marano 47A & 031538.8-551043 & 43.49 & 43.72 & 38 & $1.5 \pm 0.5 \pm 0.5$ & 2.0 & $149 / 157$ & 2.0 & $180 / 157$ \\
\hline Marano 50A & $031410.1-551746$ & 43.76 & 44.05 & 50 & $2.5 \pm 0.6 \pm 0.8$ & 2.0 & $182 / 190$ & 2.0 & 195/190 \\
\hline Marano 51A & 031630.6-551501 & 43.37 & 43.61 & 47 & $2.5 \pm 0.9 \pm 0.9^{*}$ & 2.0 & $131 / 181$ & 2.0 & $132 / 183$ \\
\hline Marano 63A & 031517.1-550602 & 44.57 & 44.81 & 29 & $4.1 \pm 1.4 \pm 1.4$ & 2.0 & $125 / 140$ & 2.0 & $127 / 140$ \\
\hline Marano 66A & $031500.8-550718$ & 43.36 & 43.45 & 39 & $0.3 \pm 0.2 \pm 0.2$ & 2.0 & $127 / 130$ & 2.0 & $129 / 130$ \\
\hline Marano 116A & 031620.9-551651 & 43.31 & 43.72 & 28 & $5.6 \pm 1.2 \pm 1.0$ & 2.0 & $129 / 126$ & 2.0 & $136 / 126$ \\
\hline Marano 133A & 031426.4-552113 & 44.04 & 44.04 & 14 & $0.0 \pm 0.8 \pm 0.0$ & 2.0 & $87 / 70$ & 2.0 & $94 / 70$ \\
\hline Marano 171A & 031351.2 & 43.61 & 44.00 & 14 & $5.4 \pm 1.5 \pm 1.0$ & 2.0 & $46 / 51$ & 2.0 & $63 / 51$ \\
\hline Marano 224B & 031304.9-551606 & 43.71 & 44.25 & 8 & $12.4 \pm 3.5 \pm 1.7$ & 2.0 & $48 / 53$ & 2.0 & $70 / 53$ \\
\hline Marano 253A & $031438.2-550648$ & 42.97 & 43.55 & 11 & $14.5 \pm 5.0 \pm 3.2$ & 2.0 & $64 / 85$ & 2.0 & $73 / 85$ \\
\hline Marano 463A & 031625.3-550839 & 44.37 & 44.53 & 7 & $1.6 \pm 1.0 \pm 0.9$ & 2.0 & $76 / 45$ & 2.0 & $81 / 45$ \\
\hline Marano 610A & $031552.0-551222$ & 43.28 & 43.75 & 17 & $10.1 \pm 3.1 \pm 2.3^{*}$ & 2.0 & $110 / 119$ & 2.0 & $113 / 120$ \\
\hline X21516_135 & 022626.7-043654 & 44.73 & 45.15 & 29 & $14.8 \pm 5.7 \pm 4.8$ & 2.0 & $61 / 79$ & 2.0 & $66 / 79$ \\
\hline X00851_154 & $221541.6-173753$ & 44.48 & 44.70 & 261 & $5.6 \pm 2.0$ & $1.6 \pm 0.1$ & $572 / 621$ & $1.8 \pm 0.6$ & $583 / 621$ \\
\hline X01135_126 & 015257.5-140839 & 43.85 & 43.85 & 392 & $0.0 \pm 0.1$ & $1.7 \pm 0.1$ & $355 / 513$ & $2.1 \pm 0.2$ & $354 / 513$ \\
\hline X03246_092 & $004345.8-202955$ & 43.48 & 43.84 & 35 & $3.8 \pm 1.2 \pm 1.3$ & 2.0 & $74 / 67$ & 2.0 & $67 / 67$ \\
\hline phl5200-001 & $222826.4-051820$ & 44.66 & 44.92 & 772 & $4.5 \pm 0.4$ & $1.6 \pm 0.1$ & $834 / 970$ & $1.2 \pm 0.2$ & $1200 / 970$ \\
\hline sds 1b-014 & 021842.9-050437 & 44.35 & 44.45 & 491 & $0.4 \pm 0.1$ & $1.8 \pm 0.1$ & $546 / 593$ & $2.0 \pm 0.2$ & $650 / 593$ \\
\hline
\end{tabular}

* - A two component fit was used, an absorbed power law $\left(N_{\mathrm{H}}\right.$ is given here) and an unabsorbed power law to model the soft excess.

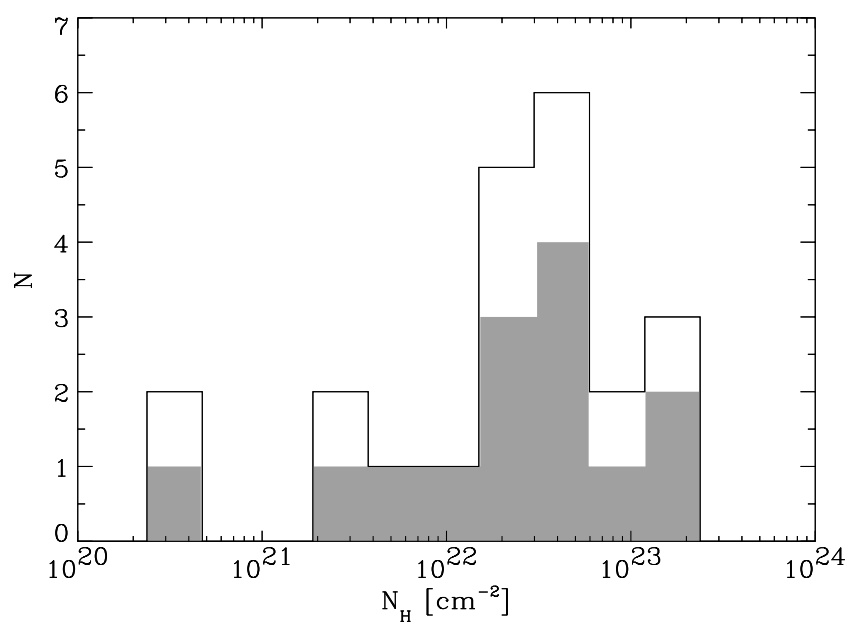

Fig. 6. Intrinsic $N_{\mathrm{H}}$ distribution of all 22 type II objects (black) and of the 14 type II QSOs (grey filled histogram).

$N_{\mathrm{H}}$ distribution of AGN and QSOs. Although a tentative anticorrelation of $N_{\mathrm{H}}$ vs. redshift below $z<1$ and a direct correlation above $z=1$ is indicated, when all data is taken together there is no significant trend in $N_{\mathrm{H}}$ with redshift. Considering the typical flux and column densities found for our sources we expected all $z>1$ type II objects to be classified as QSOs. At lower redshifts $\sim 40 \%$ of the type II QSO candidates are actually type II QSOs.

Statistical fluctuations in the X-ray spectrum can lead to high values of spuriously measured $N_{\mathrm{H}}$ values at high redshifts (e.g. Akylas et al. 2006). However, Fig. 5 show that objects with intrinsic $N_{\mathrm{H}}$ of several $10^{22}-10^{23} \mathrm{~cm}^{-2}$ are not significantly influenced by systematic trends in redshift. The fit method is also able to pick up much higher absorptions than found in our sources. The scatter in the mentioned $N_{\mathrm{H}}$ range at $z>2.5$ is consistent with an intrinsic absorption of $N_{\mathrm{H}}=10^{23} \mathrm{~cm}^{-2}$ for all sources.

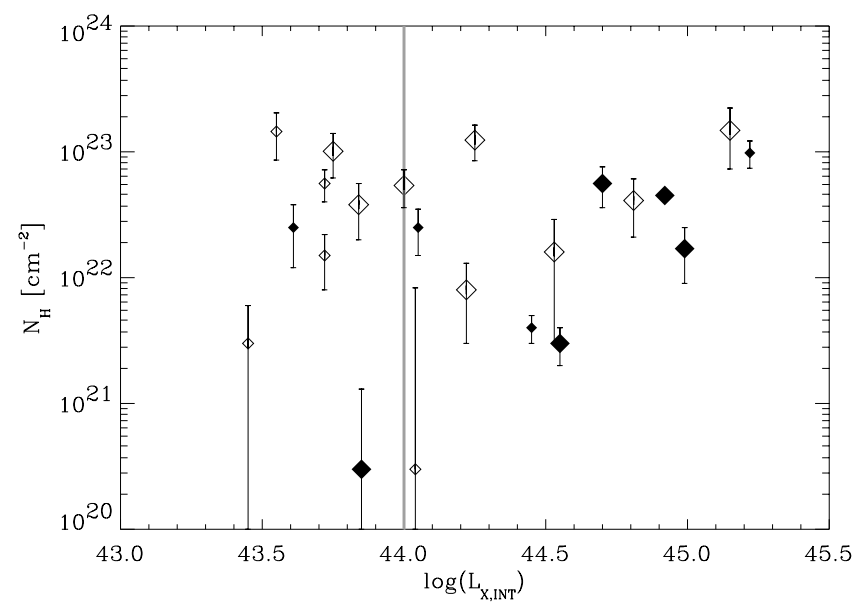

Fig. 7. Intrinsic $N_{\mathrm{H}}$ vs. de-absorbed intrinsic $0.5-10 \mathrm{keV}$ X-ray luminosity. Large symbols represent optical secure type II QSO candidates while small symbols illustrate the tentative sample. Open symbols indicate X-ray sources that have less than 40 net PN counts in the $0.2-8 \mathrm{keV}$ band; filled symbols $\geq 40$ net PN counts. The vertical solid line at $\log \left(L_{\mathrm{X}_{\mathrm{INT}}} /(\mathrm{erg} / \mathrm{s})\right)=44$ marks the dividing line between AGN and high luminosity QSOs. Objects with $\log \left(L_{\mathrm{X}_{\mathrm{INT}}} /(\mathrm{erg} / \mathrm{s})\right) \geq 44$ and $N_{\mathrm{H}} \geq 10^{22} \mathrm{~cm}^{-2}$ (upper right corner) fall in the "type II QSO region" as defined by Mainieri et al. (2002).

As a further test of the impact of statistical fluctuations, we plot in Fig. 8 the amount of $N_{\mathrm{H}}$ that is needed to reduce the $0.5 \mathrm{keV}$ flux by $30 \%$ and $50 \%$. The $30 \%$-line agrees well with the $90 \%$ contours of unabsorbed sources in Fig. 5. Consequently, even for the sources at $z>2.5$ the fitted $N_{\mathrm{H}}$ values are unlikely to be caused by statistical fluctuations.

The previous conclusions depend on not having misinterpreted Compton-thick absorbed objects $\left(N_{\mathrm{H}}>1.5 \times 10^{24} \mathrm{~cm}^{-2}\right)$ as moderately absorbed objects. Increasing column densities 


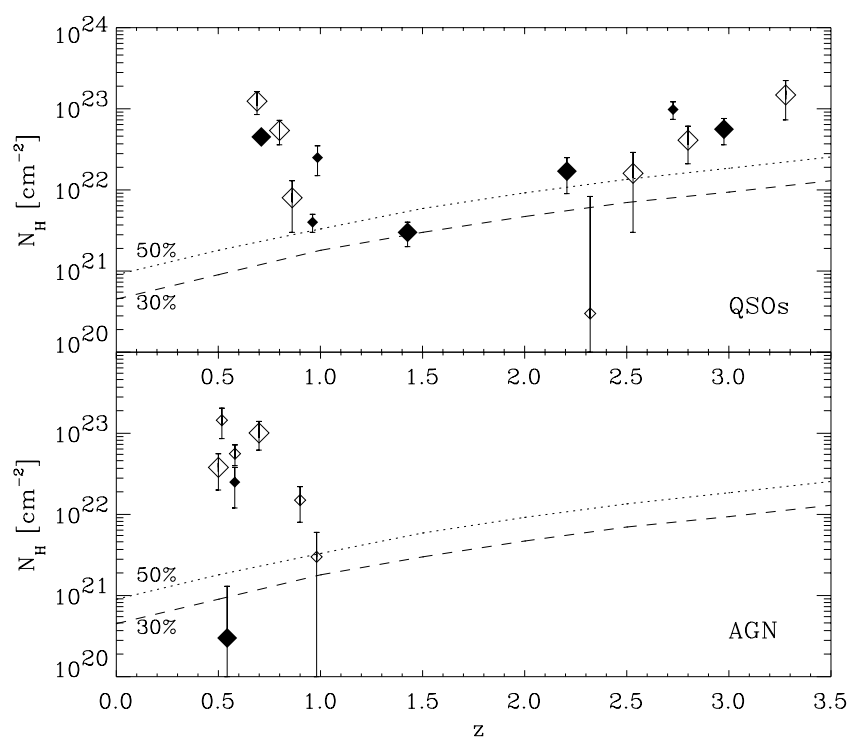

Fig. 8. Intrinsic $N_{\mathrm{H}}$ vs. redshift diagram for type II QSOs (top panel) and type II AGN (lower panel). Labels as in Fig. 7. The dashed and dotted lines represent the $N_{\mathrm{H}}$ values which correspond to a $30 \%$ and $50 \%$ decrease in the $0.5 \mathrm{keV}$ flux at the given redshift, respectively.

cause a hardening of the X-ray spectrum. This trend stops when the material becomes optically thick to electron scattering. The $\mathrm{X}$-ray spectrum of a Compton-thick absorbed source is completely dominated by the reflection component. Their X-ray spectra show soft X-ray radiation but with a much lower photon index ( $\Gamma \sim-0.4-1.3$, Maiolino et al. 1998; Bassani et al. 1999; Risaliti et al. 2000; Iwasawa et al. 2001; Comastri 2004). We simulated an X-ray spectrum with $\Gamma=1.0$ as observed in the 3-12 keV band for NGC 1068. When we fit the spectrum with a fixed $\Gamma=2.0$, we recover values for absorption of $N_{\mathrm{H}}=0.7 \times 10^{22} \mathrm{~cm}^{-2}, N_{\mathrm{H}}=1.9 \times 10^{22} \mathrm{~cm}^{-2}$ and $N_{\mathrm{H}}=$ $3.8 \times 10^{22} \mathrm{~cm}^{-2}$ for redshifts of $z=1,2,3$ respectively. Hence, moderately absorbed sources are found if intrinsically Comptonthick absorbed sources are studied. However, we are able to exclude a general misinterpretation of the spectra based on the following arguments.

First we tested if the X-ray spectra are better modelled with a purely reflection-dominated model (pexrav). We used solar abundances and a cutoff energy of $100 \mathrm{keV}$. The ratio between the reflected and direct component (fit parameter: scaling factor) was set to 100 in order to obtain pure reflection. The cosine of the inclination was left as a free parameter. Again, we fitted the photon index as a free parameter only in the case the X-ray source had more than $100 \mathrm{PN}$ net counts.

Only the data of X-ray source X01135_126 and X03246_092 are slightly better represented by the reflection model in comparison to a single power law with intrinsic absorption. Further four X-ray sources (Marano 51A, 63A, 66A, 610A) can be equally well modelled with a reflection model or a power law with intrinsic absorption (see Table 4). However, most of these objects have PN net counts of $<50$. Only X-ray source X01135_126 has $N_{\text {PN-Counts }}>100$. Low count spectra have the obvious problem that their X-ray data can be well fitted by different models. Nevertheless, the majority of our objects is only adequately fitted by a model that includes intrinsic absorption.

Secondly the reflected component of Compton-thick absorbed sources is $\sim 50-150$ times weaker than the deabsorbed intrinsic X-ray luminosity (Brandt \& Hasinger 2005). Hence they are usually found at low values of $f_{\mathrm{X}} / f_{\text {[OIII] }}$

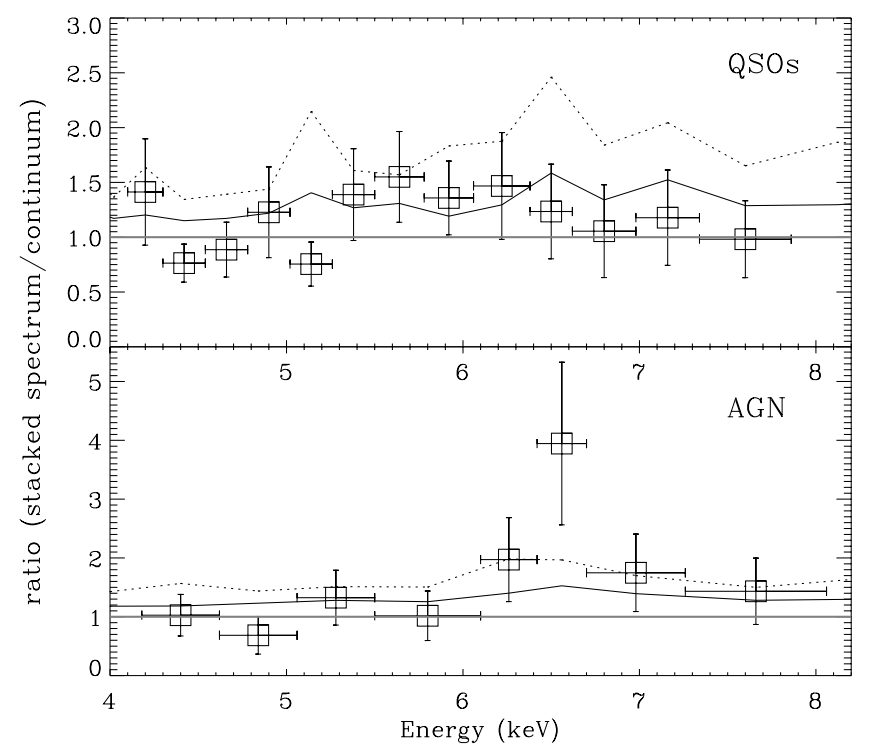

Fig. 9. Stacked X-ray spectrum of all 14 type II QSOs (upper panel) and 8 type II AGN (lower panel). The QSOs stack contains $\sim 1600$ counts in the shown 4-8.2 keV energy range, the AGN stack 300 counts. The squares represent the ratio between the flux of the stacked spectrum and the simulated continuum spectrum. The solid line shows the $1 \sigma$ detection error while the dotted line represents the $2 \sigma$ detection error. The solid grey line at a ratio of one illustrates the continuum flux.

(Bassani et al. 1999). For the majority of our objects the [O III] line is redshifted out of our spectral range. As a second best estimator we adopt $f_{\mathrm{X}} / f_{\text {OPT }}$ rather than $f_{\mathrm{X}} / f_{\text {[OIII] }}$. Figure 2 shows that almost all objects have high $f_{\mathrm{X}} / f_{\mathrm{OPT}}$ which is inconsistent with Compton-thick absorbed objects. Only X00851_154 has a rather low $f_{\mathrm{X}} / f_{\mathrm{OPT}}$ ratio, but the best X-ray spectral fit is a moderately absorbed power law with $\Gamma=1.6$. Under the assumption of Compton-thick absorption, all studied objects would be classified as QSOs and our sample would contain the most X-ray luminous QSOs ever studied $\left(L_{\mathrm{X}_{\mathrm{INT}}} \sim 10^{47} \mathrm{erg} / \mathrm{s}\right)$.

\subsection{Stacking of $X$-ray spectra}

Another indication for Compton-thick absorption is the detection of the iron line which is normally outshone by the direct component. Due to the reflection of the X-ray radiation from cold material in the torus, an iron $\mathrm{K} \alpha$ fluorescence line with an equivalent width $E W \gtrsim 1 \mathrm{keV}$ is expected (Turner et al. 1997; Risaliti 2002).

The low count numbers in our X-ray spectra do not allow the study of individual spectral features such as an iron line. Based on the assumption that the intrinsic X-ray properties in the sample are similar, a stack of the individual spectra can reveal the presence of an iron line. Since the number of individual X-ray spectra increases the SNR in the stacked spectrum, we decided to include all 14 type II QSOs regardless of the optical flag. The assumption is justified because the secure and tentative sample do not show differences in $f_{\mathrm{X}} / f_{\mathrm{OPT}}$ (Fig. 2) and absorption values (Fig. 6).

Corral et al. (2008) explain the stacking process in detail. Here we outline the essential components of the process. Using the best-fit parameters, unfolded spectra (eufspec in XSPEC) from the ungrouped observed X-ray spectra (MOS and PN data) are extracted. The unfolded spectra are corrected for Galactic foreground absorption, shifted to the rest-frame and normalised to the same rest-frame flux in the $2-5 \mathrm{keV}$ band. The flux is 
rebinned into a common energy grid for all spectra. The stacked spectrum is binned to a final energy grid that contains at least 100 counts per energy bin. The final errors of the stacked spectrum are based on Gaussian propagation of the errors in the individual spectra. To distinguish between real spectral features and artifacts from the averaging process the underlying continuum has to be modelled. Each source was simulated 100 times using the same model (absorption plus powerlaw) with the same $2-8 \mathrm{keV}$ flux as observed in the real spectra. The individual simulated X-ray continuum spectra from all different X-ray sources are stacked exactly as the observed X-ray spectra which are used to determine the $1 \sigma$ and $2 \sigma$ contours $(68 \%$ and $95 \%$ of all simulated continuum spectra).

The $0.5-10 \mathrm{keV}$ final stacked spectrum of the QSOs contains $\sim 4300$ counts. Together with the $1 \sigma$ and $2 \sigma$ contours the ratio of the observed spectrum to the average simulated continuum is shown in Fig. 9, upper panel. The stacked type II QSO spectrum shows no residuals around 6-7 keV which would correspond to an iron $\mathrm{K} \alpha$ emission line. The SNR in the stacked spectrum is sufficient to detect an iron line with $E W \gtrsim 1 \mathrm{keV}$. The stacked spectrum does not provide any evidence for Compton-thick absorbed sources based on a line emission and supports the assumption that our type II QSOs are actually moderately absorbed sources.

Interestingly, the stacked spectrum of our 8 type II AGN does show a very prominent emission line (Fig. 9, lower panel), although in none of the individual AGN X-ray spectra a significant excess at $6-7 \mathrm{keV}$ is recognised (see the X-ray spectra in the Appendix A). Since only a few objects are included in the AGN stack we used energy bins containing 30 counts instead of 100 counts. We fitted the positive residuals in the stacked AGN spectrum with a Gaussian line profile. A line is detected with a significance above $2 \sigma$ at a line energy of $E=6.6_{-0.08}^{+0.07} \mathrm{keV}$ with a $\sigma=230_{-40}^{+100} \mathrm{eV}$. The equivalent width of the fit is $E W=$ $2.0_{-0.7}^{+0.6} \mathrm{keV}$.

In principle there are two possible explanations for the detection of a broad iron fluorescence line.

- a reflection line from the accretion disk, which can be significantly broadened due to relativistic effects;

- a blend of narrow lines that is observed as a single unresolved broad line due to the low signal-to-noise ratio of our observations.

The typical equivalent widths of the disk iron lines are usually significantly lower than the $E W$ of the iron line found in our AGN stack. The energy and width of the line is more consistent with a blend of narrow lines. Depending on the properties of the reflecting material different ionisation stages of iron are excited as observed in the the Compton-thick absorbed object NGC 1068 (Ogle et al. 2003). If the signal-to-noise ratio is lowered the three resolved lines will be observed as an unresolved broad $(E W \gtrsim 1 \mathrm{keV})$ line with a peak above $6.4 \mathrm{keV}$. In this case a significant number of objects that we classified as AGN could be Compton-thick absorbed. Interestingly, except for one object, all X-ray sources, that can be better or equally well fitted by a purely reflection-dominated model in comparison to the power law plus intrinsic absorption model, are AGN.

\section{Conclusions}

We selected 51 spectroscopically classified type II objects from the XMM-Newton Marano field survey, XWAS, and AXIS. We re-investigate the optical spectra for narrow and common AGN high excitation lines, as well as verifying the X-ray counterpart determination. 22 sources meet our selection criteria (13 secure identification, 9 tentative). All sources have $z \geq 0.5$ and observed 0.5-10 keV X-ray luminosities (not corrected for intrinsic absorption) of $L_{\mathrm{X}_{\mathrm{OBS}}} \sim 10^{43-45} \mathrm{erg} / \mathrm{s}$. The sample is not flux limited.

The selection of both optical, narrow high excitation emission lines and intrinsic X-ray luminosities $L_{\mathrm{X}_{\mathrm{INT}}} \geq 10^{44} \mathrm{erg} / \mathrm{s}$ yielded 14 type II QSOs.

Since the distribution of net PN counts peaks at $\sim 40$, the $\mathrm{X}$-ray spectral analysis has to account for this very low numbers of counts. We extensively simulated and studied such low count number X-ray spectra. A binning with at least one count per bin combined with the Cash-statistic recovered the input values best. We proved that the method is able to find absorption up to $N_{\mathrm{H}}=$ $10^{24} \mathrm{~cm}^{-2}$.

However, we discover only moderately absorbed type II QSOs. One QSO is consistent with being unabsorbed but it belongs to the tentative sample. Compton-thick absorbed sources may be detected as moderately absorbed but we can exclude this scenario for the majority of our sources. The X-ray data are not well fitted by reflection models. The $f_{\mathrm{X}} / f_{\mathrm{OPT}}$ values of the objects and the non-detection of a broad iron line in the stacked type II QSOs spectrum give evidence that we have not misclassified Compton-thick absorbed sources. In contrary to the QSO stack, the stack of 8 type II AGN revealed a very prominent iron line with an $E W \sim 2 \mathrm{keV}$. However, the shape of the single X-ray spectra and the modelling of the X-ray data showed that the majority of our AGN are most probably moderately absorbed X-ray sources.

The column density distribution found by us agrees well with those in deep Chandra and XMM-Newton surveys (Mainieri et al. 2002; Szokoly et al. 2004; Mateos et al. 2005; Ptak et al. 2006). These authors also reported a few cases of unabsorbed type II AGNs, as well as evidence for some heavily absorbed sources $\left(N_{\mathrm{H}} \sim 10^{24} \mathrm{~cm}^{-2}\right)$. La Franca et al. (2005) studied column density trends with X-ray luminosity in different redshift bins. They classified type II AGN as all sources that do not show any emission lines with $F W H M>2000 \mathrm{~km} \mathrm{~s}^{-1}$. Independent of $\mathrm{X}$-ray luminosity and redshift bins, they discovered type II AGN with an average absorption of $N_{\mathrm{H}} \sim 10^{23} \mathrm{~cm}^{-2}$, slightly above the column density peak of our survey $\left(N_{\mathrm{H}}=4 \times 10^{22} \mathrm{~cm}^{-2}\right)$. The present study of strictly classified type II AGN/QSOs, based on the optical spectra, verified that there is no obvious trend of absorbing column density with redshift or X-ray luminosity.

Our results apparently contradict studies of the local universe. Bassani et al. (1999) and Risaliti et al. (2000) found 75\% of their sources with high absorption $\left(N_{\mathrm{H}}>10^{23} \mathrm{~cm}^{-2}\right)$ and $25-45 \%$ with $N_{\mathrm{H}}>10^{24} \mathrm{~cm}^{-2}$. Either the column density properties change dramatically from the local to distant universe or the majority of the heavily absorbed distant sources are missed in our surveys. Indeed even the most luminous Compton-thick absorbed sources in the local universe, e.g. NGC 6240, could not have been detected by our survey if they were at $z \gtrsim 0.4$.

In summary, the column densities in our survey show no trend in X-ray luminosity and no clear trend in redshift. If we compare our results with samples of AGN in the local universe, our sample does not contain a significant fraction of heavily absorbed sources $\left(N_{\mathrm{H}}>10^{23} \mathrm{~cm}^{-2}\right)$. Our survey of objects with $z \geq 0.5$ is limited to observed X-ray luminosities in excess of $L_{\mathrm{X}_{\mathrm{OBS}}}>10^{43} \mathrm{erg} / \mathrm{s}$. We can only expect to find QSOs intrinsically more luminous than $L_{\mathrm{X}_{\mathrm{INT}}}>10^{45} \mathrm{erg} / \mathrm{s}$, if they are Compton-thick absorbed. Norman et al. (2002) claim that for the most distant type II QSO ever detected $(z=3.7)$, there is strong evidence for heavy or even Compton-thick absorption. 
However, our survey rules out large numbers of Compton-thick absorbed sources with X-ray luminosities of $L_{\mathrm{X}_{\mathrm{INT}}}>10^{45} \mathrm{erg} / \mathrm{s}$. Hence, potential Compton-thick absorbed objects at high redshifts are likely to have similar X-ray luminosities to Comptonthick absorbed objects in the local universe. In order to find a supposed, rare population of very luminous, Compton-thick absorbed QSOs a larger survey area is needed. The 2XMM catalogue (Watson et al. 2008) with a survey area of $\sim 360 \mathrm{deg}^{2}$ could provide a valuable source to reveal such a population.

Acknowledgements. Mirko Krumpe is supported by the Deutsches Zentrum für Luft- und Raumfahrt (DLR) GmbH under contract No. FKZ 50 OR 0404 Georg Lamer acknowledges support by the Deutsches Zentrum für Luft- und Raumfahrt (DLR) GmbH under contract no. FKZ 50 OX 0201. Amalia Corral, Francisco J. Carrera, and Xavier Barcons acknowledge financial support by the Spanish Ministry of Education and Science, through projects ESP2006-13608C02-01. M.P., S.M., J.T. and M.W. thank STFC for financial support.

\section{References}

Akiyama, et al. 2008, in preparation

Akylas, A., Georgantopoulos, I., Georgakakis, A., Kitsionas, S., \& Hatziminaoglou, E. 2006, A\&A, 459, 693

Antonucci, R. 1993, ARA\&A, 31, 473

Arnaud, K. A. 1996, Astronomical Data Analysis and Systems V, ed. G. Jacoby, \& J. Barnes, ASP Conf. Ser., 101, 17

Barcons, X., Carrera, F. J., Watson, M. G., et al. 2002, A\&A, 382, 522

Barcons, X., Carrera, F. J., Ceballos, M. T., et al. 2007, A\&A, 476, 119

Barger, A. J., Cowie, L. L., Brandt, W. N., et al. 2002, AJ, 124, 1839

Bassani, L., Dadina, M., Maiolino, R., et al. 1999, ApJS, 121, 473

Brandt, W. N., \& Hasinger, G. 2005, ARA\&A, 43, 827

Carrera, F. J., Ebrero, J., Mateos, S., et al. 2007, A\&A, 469, 27
Comastri, A. 2004 [arXiv: astro-ph/0403693]

Corral, A., et al. 2008, in preparation

Dwelly, T., \& Page, M. J. 2006, MNRAS, 372, 1755

La Franca, F., Fiore, F., Comastri, A., et al. 2005, ApJ, 635, 864

Gabriel, C., Denby, M., Fyfe, D. J., et al. 2004, Astronomical Data Analysis Software and Systems XIII, ed. F. Ochsenbein, M. Allen, \& D. Egret, ASP Conf. Ser., 314, 759

Gilli, R., Comastri, A., \& Hasinger, G. 2007, A\&A, 463, 79

Hao, L., Strauss, M. A., Tremonti, C. A., et al. 2005, AJ, 129, 1783

Hambly, N. C., MacGilivray, H. T., Read, M. A., et al. 2001, MNRAS, 326, 12791

Iwasawa, K., Matt, G., Fabian, A. C., et al. 2001, MNRAS, 326, 119

Kleinmann, S. G., Hamilton, D., Keel, W. C., et al. 1988, ApJ, 328, 161

Krumpe, M., Lamer, G., Schwope, A. D., et al. 2007, A\&A, 466, 41

Mainieri, V., Bergeron, J., Hasinger, G., et al. 2002 A\&A, 393, 425

Maiolino, R., Salvati, M., Bassani, L., et al. 1998, A\&A, 338, 781

Martinez-Sansigre, A., Rawlings, S., Bonfield, D. G., et al. 2007, MNRAS, 379,6

Mateos, S., Barcons, X., Carrera, F. J., et al. 2005, A\&A, 444, 79

Norman, C., Hasinger, G., Giacconi, R., et al. 2002, ApJ, 571, 218

Ogle, P. M., Brookings, T., Canizares, C. R., Lee, J. C., \& Marshall, H. L. 2003, A\&A, 402, 849

Page, M. J., Davis, S. W., \& Salvi, N. J. 2003, MNRAS, 343, 1241

Ptak, A., Zakamska, N. L., Strauss, M. A., et al. 2006, ApJ, 637, 147

Risaliti, G. 2002, A\&A, 386, 379

Risaliti, G., Gilli, R., Maiolino, R., \& Salvati, M. 2000, A\&A, 357, 13

Sutherland, W., \& Saunders, W. 1992, MNRAS, 259, 413

Szokoly, G. P., Bergeron, J., Hasinger, G., et al. 2004 ApJS, 155, 271

Tedds, J. A., et al. 2008, in preparation

Turner, T. J., George, I. M., Nandra, K., \& Mushotzky, R. F. 1997, ApJS, 113, 23

Tozzi, P., Gilli, R., Mainieri, V., et al. 2006, A\&A, 451, 457

Watson, M. G., et al. 2008, in preparation

Zakamska, N. L., Strauss, M. A., Krolik, J. H., et al. 2003, AJ, 126, 2125

Zombeck, M. V. 1990, Handbook of Astronomy (Cambridge University Press) 


\section{Appendix A: Optical and X-ray spectra, confidence contours}

In this section we show the optical and X-ray spectra, as well as the confidence contours of X-ray spectral fits for the parameters $N_{\mathrm{H}}$ and $\Gamma$.

Optical spectra: Optical atmospheric absorption corrected, wavelength and flux calibrated spectrum for the optical X-ray counterpart is shown. All spectra are in flux units of $10^{-18} \mathrm{erg} \mathrm{cm}^{-2} \mathrm{~s}^{-1} \AA^{-1}$. The black solid line represents the spectrum, the green solid line the error spectrum (not available in all spectra). Red markers indicate possible emission lines, blue markers absorption lines. There are exceptions in a few spectra. Spectral features at $5580 \AA$ are spurious due to incomplete subtraction of a night sky line.

\section{$X$-ray spectra:}

The X-ray spectra show the PN (black) and combined MOS (red) data, as well as the best fit model (foreground Galactic absorption, power law with intrinsic absorption at the object's redshift). In the case of Marano 51A and Marano 610A the shown best fit model consists of foreground Galactic absorption, an unabsorbed power law, and an power law with intrinsic absorption at the object's redshift to account for the soft excess in the X-ray data.

For objects that have less than $100 \mathrm{PN}$ source counts in the $0.2-8 \mathrm{keV}$ band we fixed the photon index $(\Gamma=2)$. Otherwise, free fits in $N_{\mathrm{H}}$ and $\Gamma$ are shown. The fit parameters are given in Table 4. For illustration purposes the X-ray data have been rebinned to different signal-to-noise ratio, after grouping to a minimum of one count per bin (min 1).

Confidence contours: Confidence contours of the absorbing column density in units of $N_{\mathrm{H}}=10^{22} \mathrm{~cm}^{-2}$ vs. photon index $\Gamma$ of the X-ray spectral fits. Confidence contours are plotted for 68\% (black), 90\% (red) and 99\% (green). The contours are based on free $N_{\mathrm{H}}$ and $\Gamma \mathrm{X}$-ray spectral fits for all objects independent of PN net counts.

Comments: Below every set of optical \& X-ray spectra and X-ray contour a comment for the objects shown is given. This comment includes the classification number of the optical counterpart and the corresponding redshift. Furthermore, a short fit description is given. For more details on the properties of the sources and the X-ray spectral fits see Tables 1, 4.
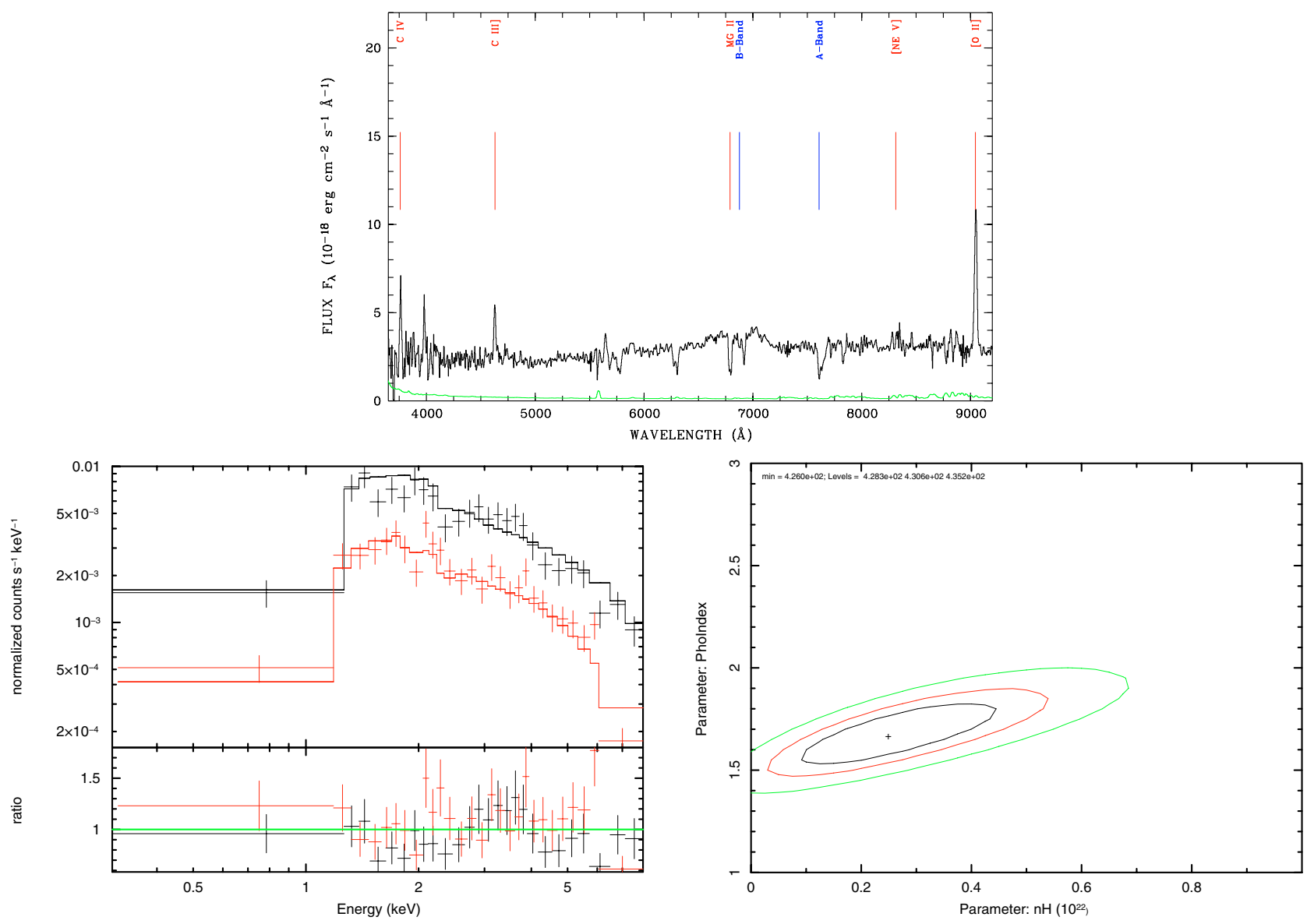

object Marano 9A, $z=1.427$, free X-ray spectrum fit. 
M. Krumpe et al.: X-ray absorption in distant type II QSOs, Online Material $p 2$
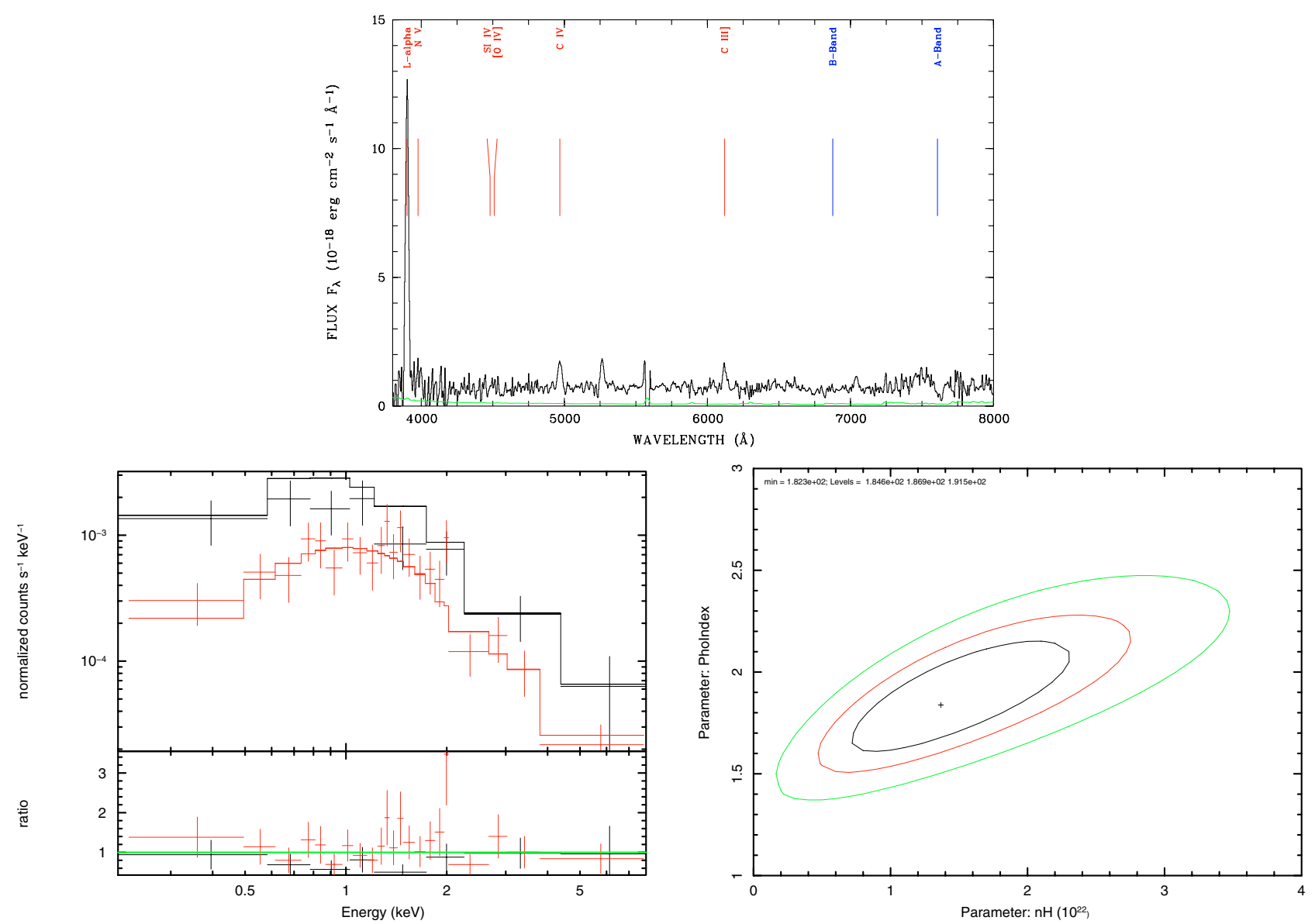

object Marano 20A, $z=2.207$, frozen $\Gamma=2.0$ X-ray spectrum fit.
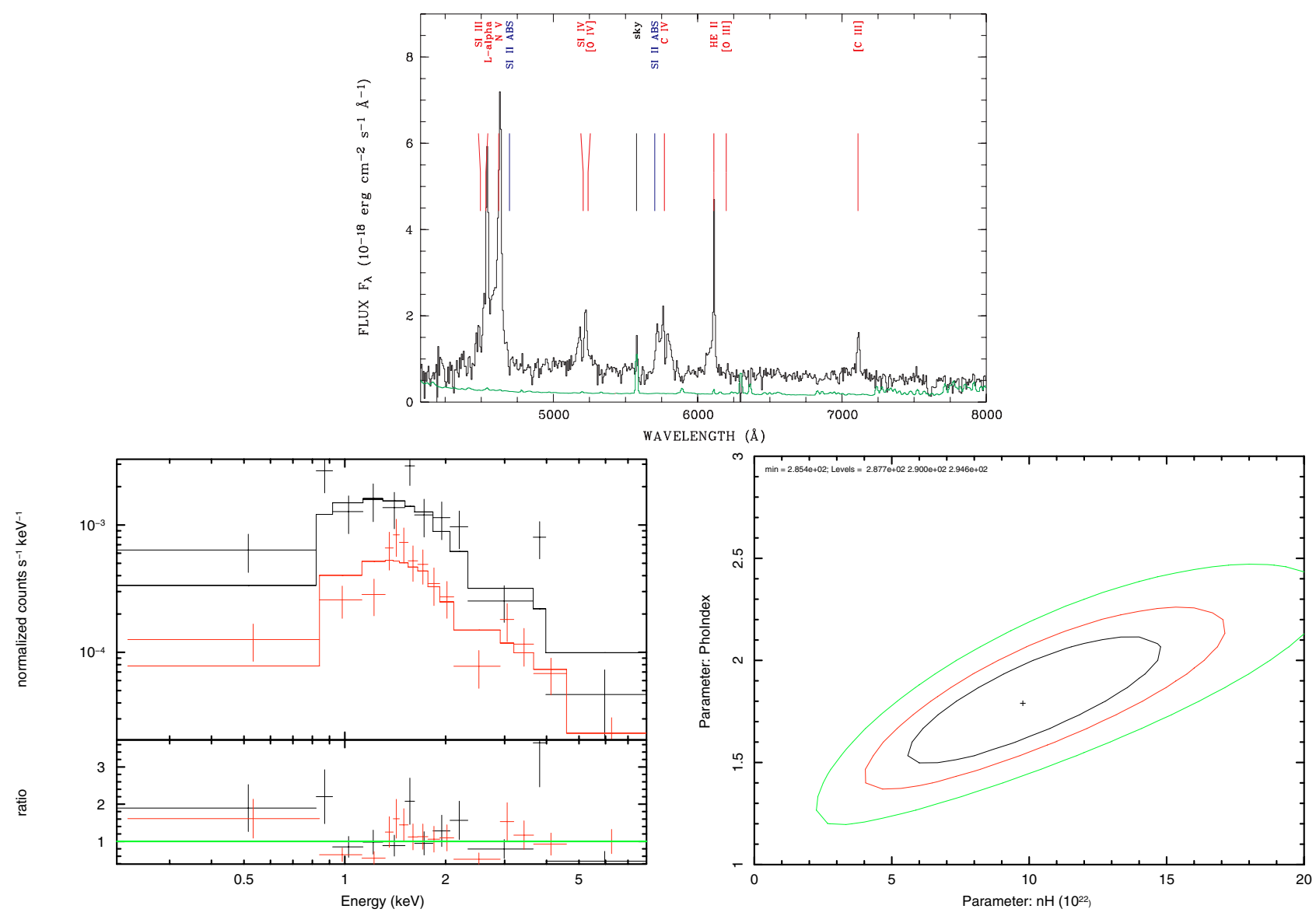

object Marano 32A, $z=2.727$, free X-ray spectrum fit. 
M. Krumpe et al.: X-ray absorption in distant type II QSOs, Online Material p 3
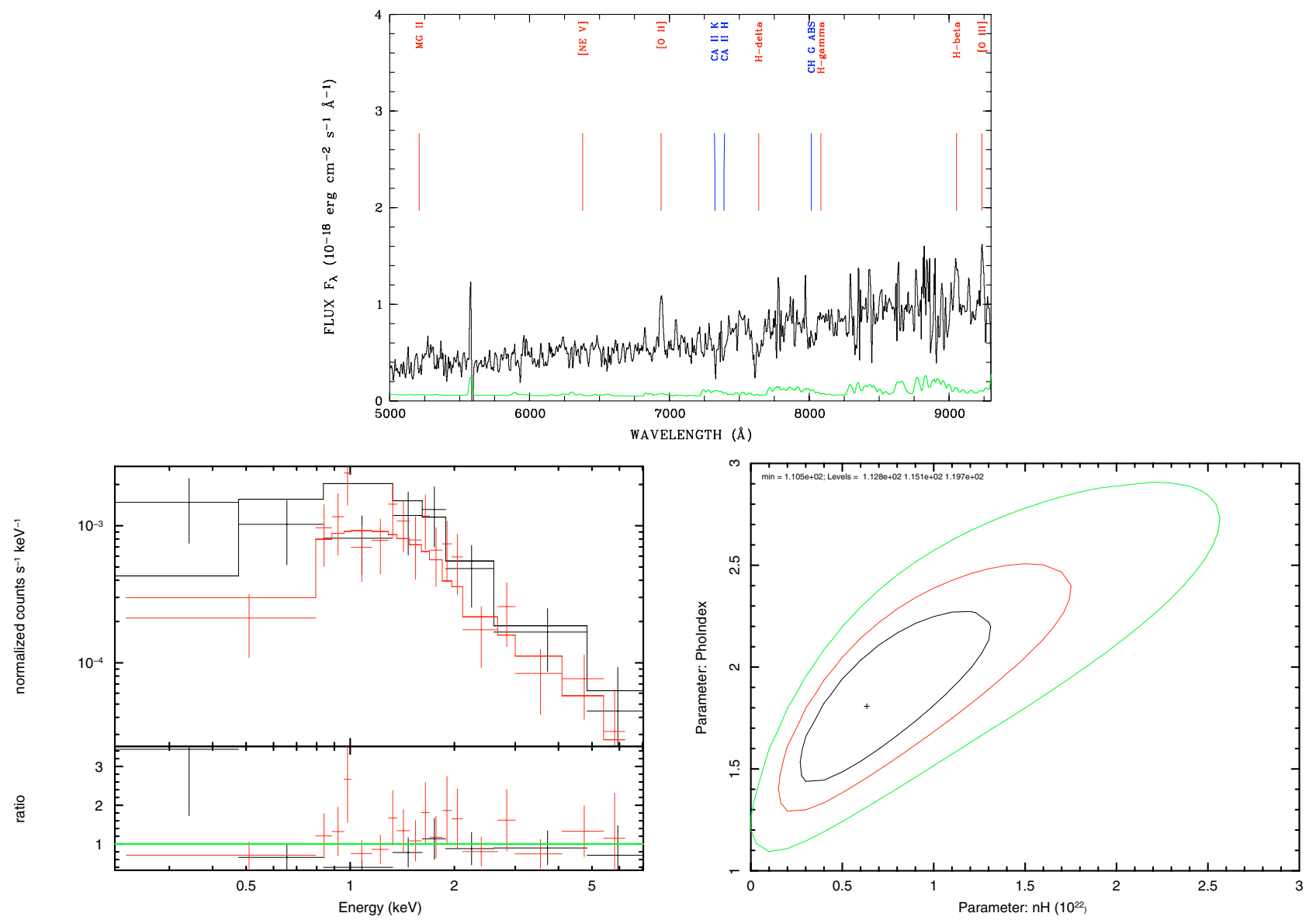

object Marano 39A, $z=0.862$, frozen $\Gamma=2.0$ X-ray spectrum fit.
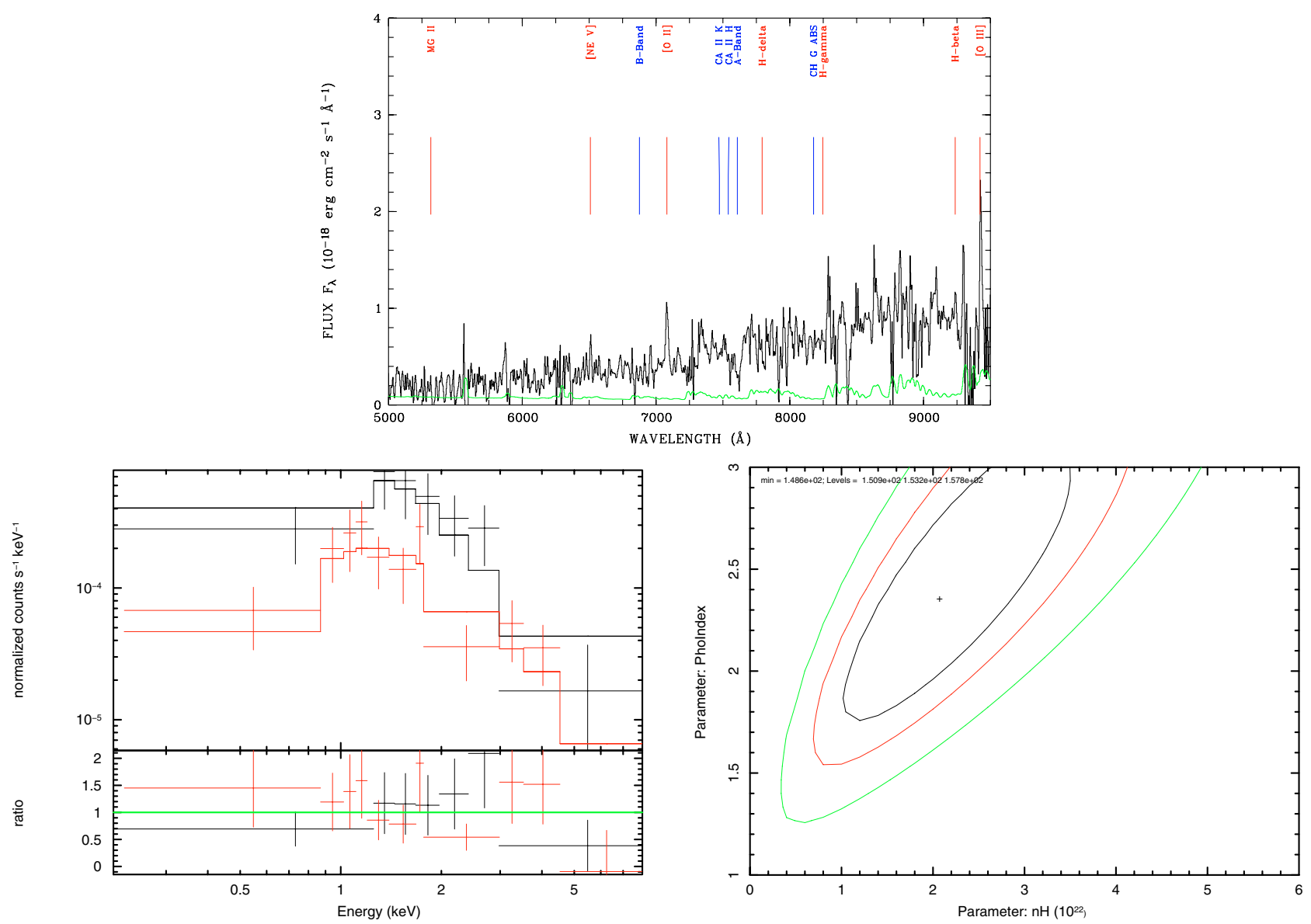

object Marano 47A, $z=0.900$, frozen $\Gamma=2.0$ X-ray spectrum fit. 
M. Krumpe et al.: X-ray absorption in distant type II QSOs, Online Material $p 4$
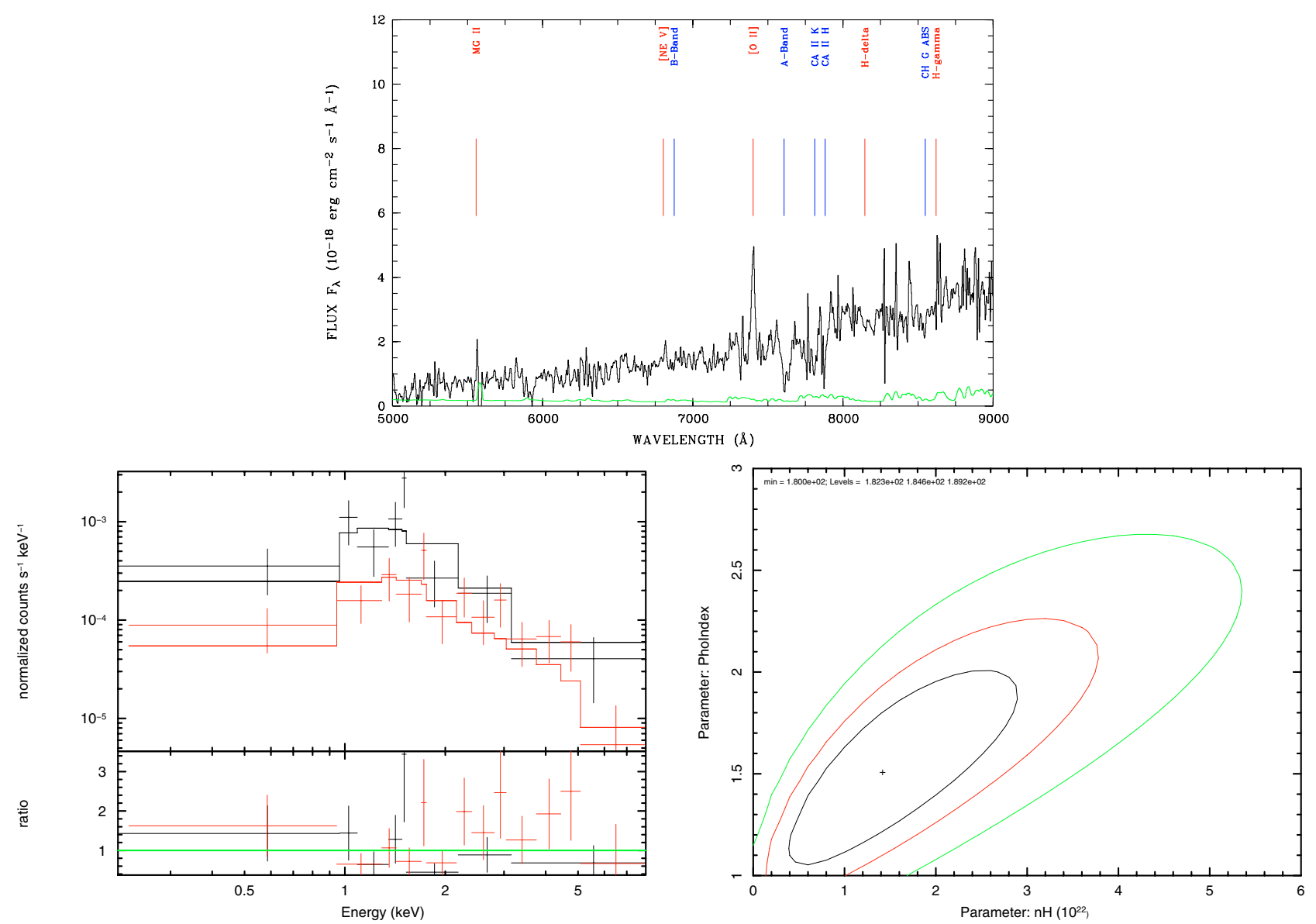

object Marano 50A, $z=0.986$, frozen $\Gamma=2.0$ X-ray spectrum fit.
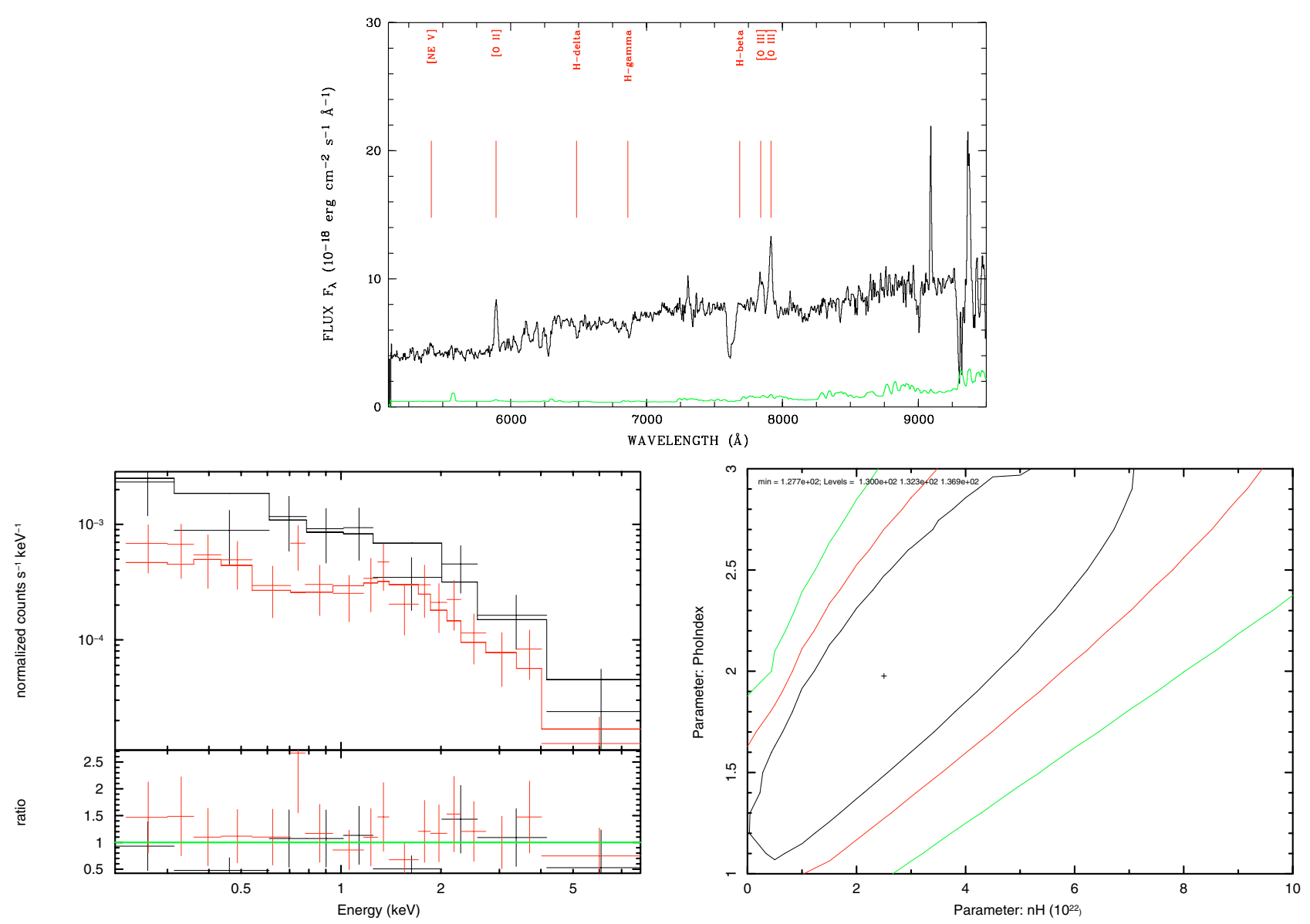

object Marano 51A, $z=0.58$, two power laws, frozen $\Gamma=2.0$ X-ray spectrum fit. 
M. Krumpe et al.: X-ray absorption in distant type II QSOs, Online Material p 5
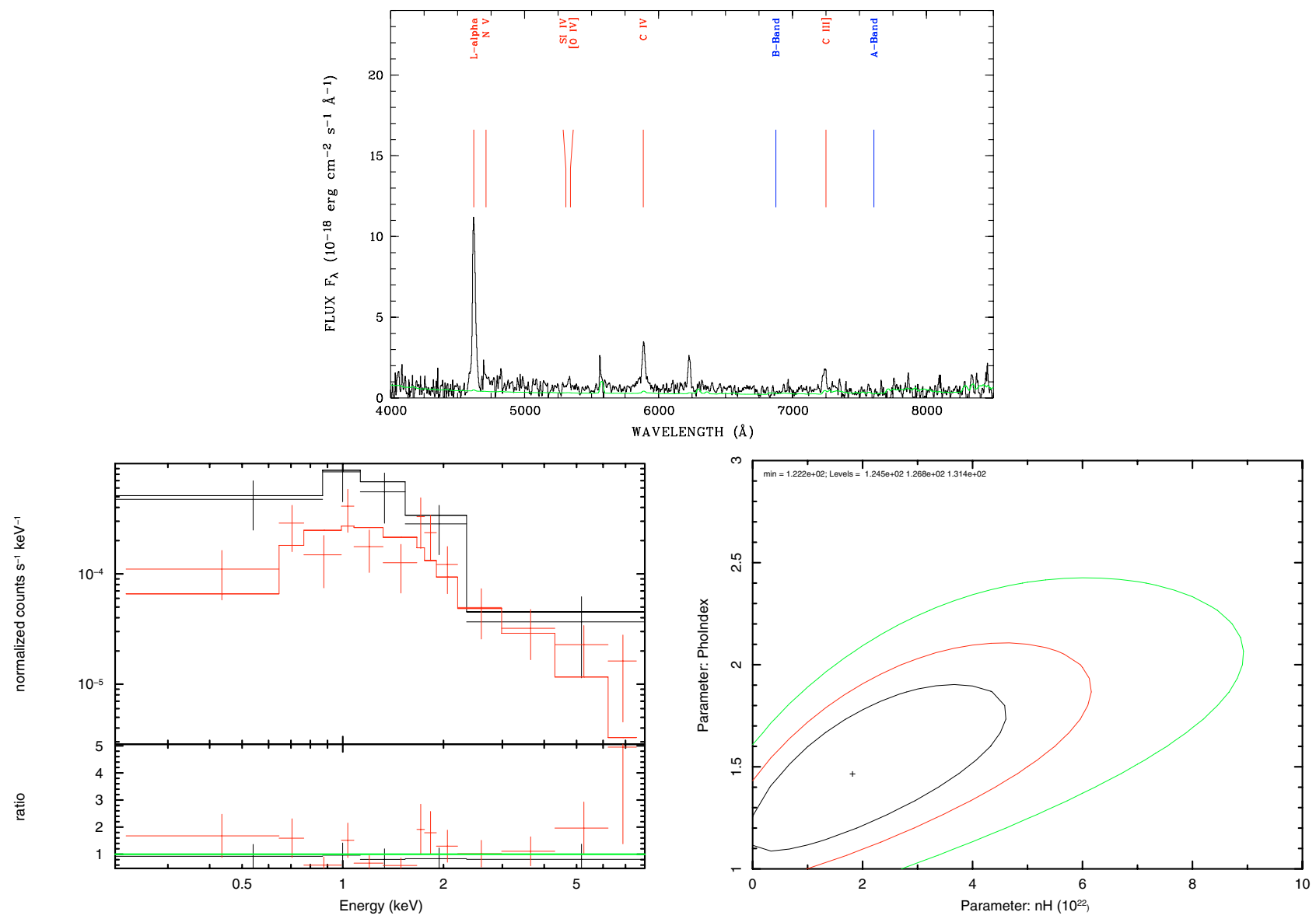

object Marano 63A, $z=2.800$, frozen $\Gamma=2.0$ X-ray spectrum fit.
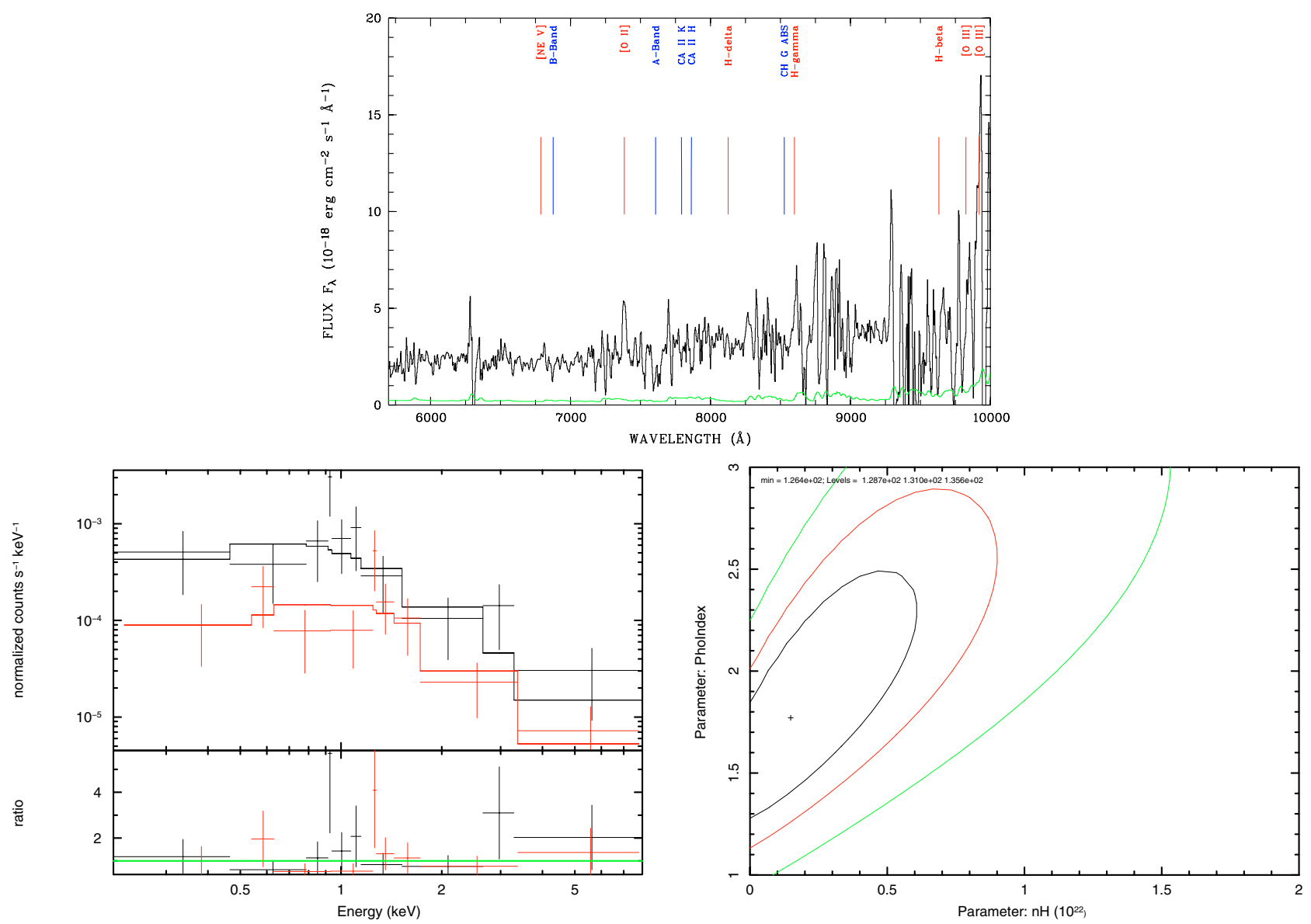

object Marano 66A, $z=0.981$, frozen $\Gamma=2.0$ X-ray spectrum fit. 
M. Krumpe et al.: X-ray absorption in distant type II QSOs, Online Material $p 6$
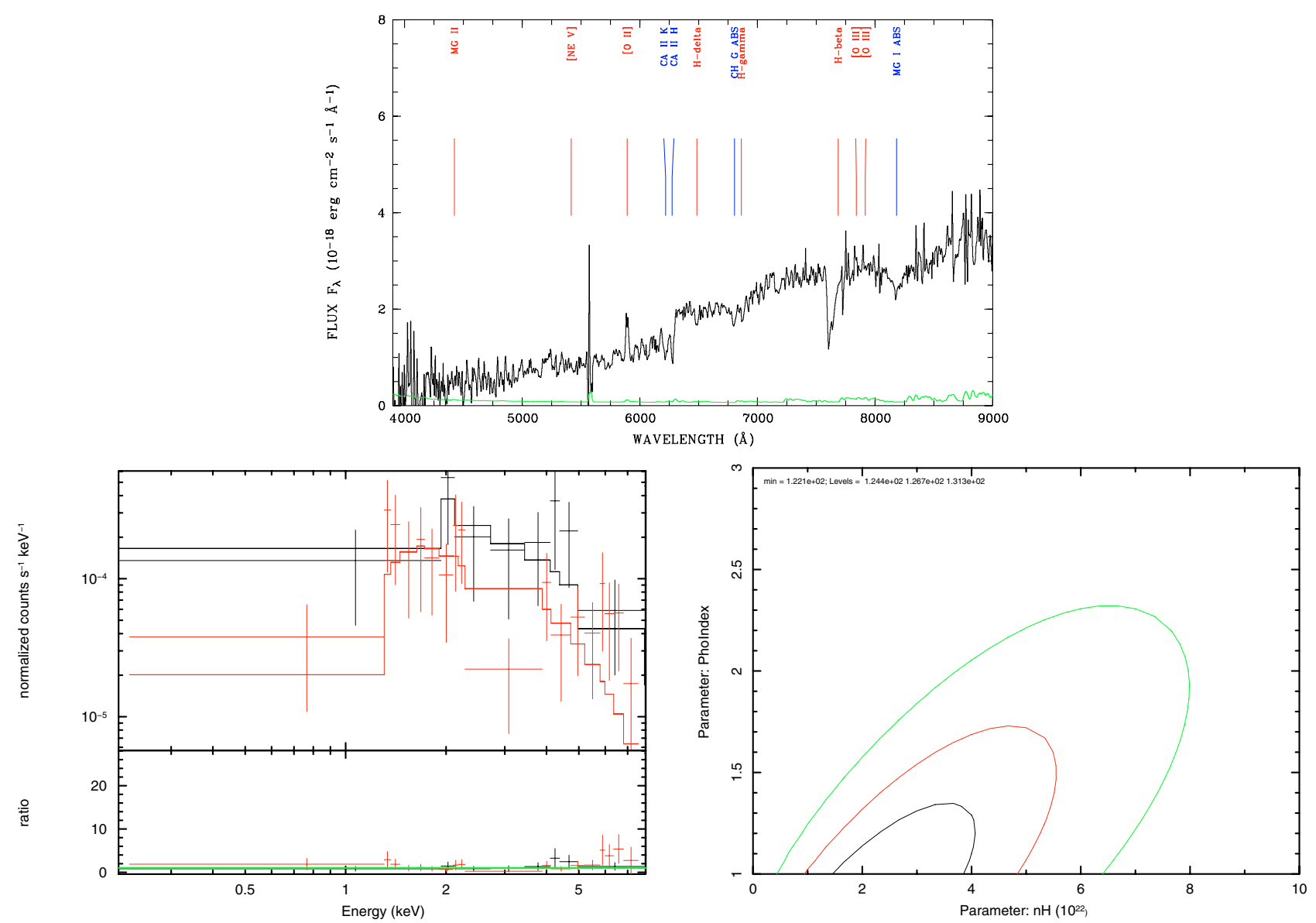

object Marano 116A, $z=0.581$, frozen $\Gamma=2.0$ X-ray spectrum fit.
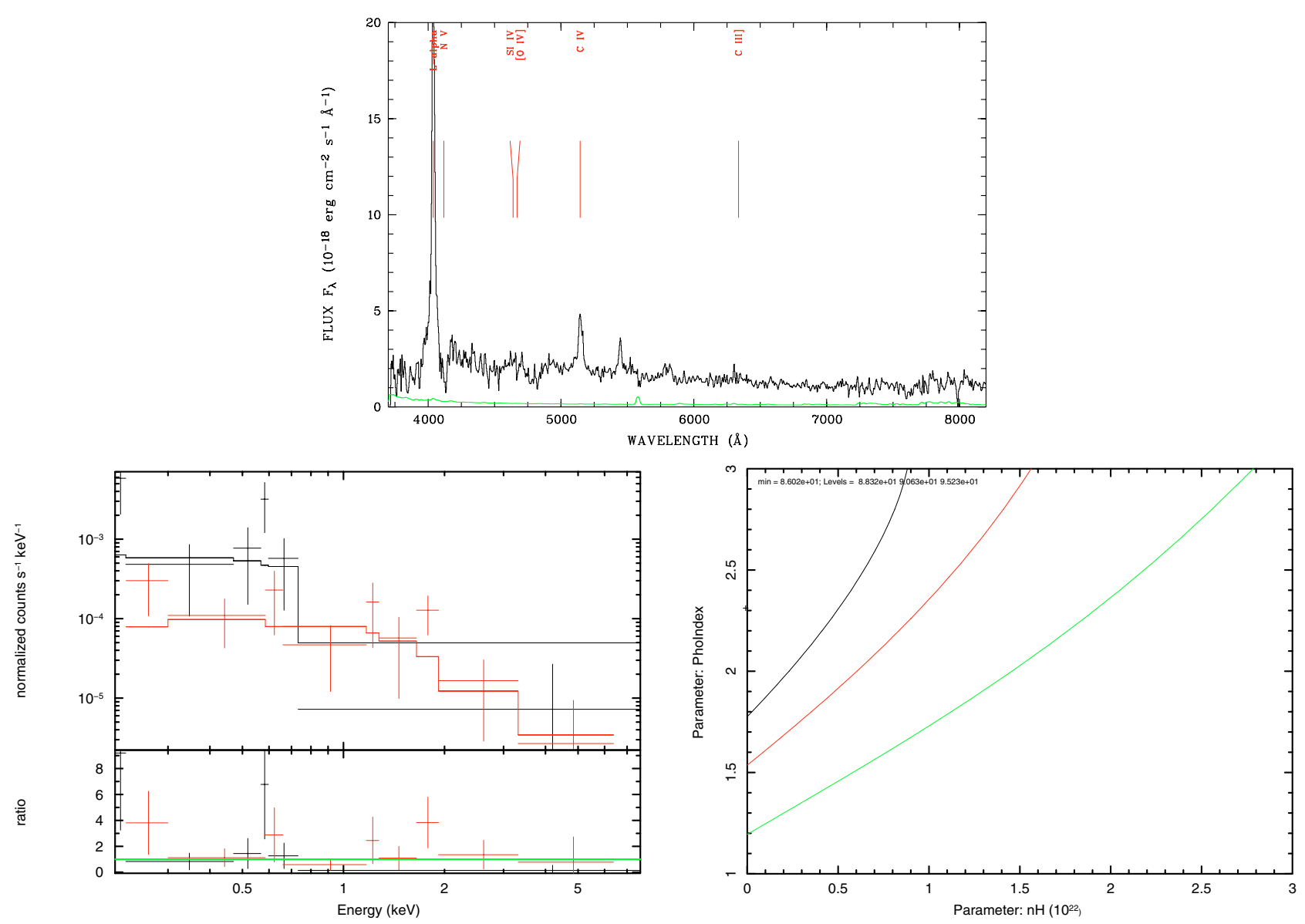

object Marano 133A, $z=2.321$, frozen $\Gamma=2.0$ X-ray spectrum fit. 
M. Krumpe et al.: X-ray absorption in distant type II QSOs, Online Material $p 7$
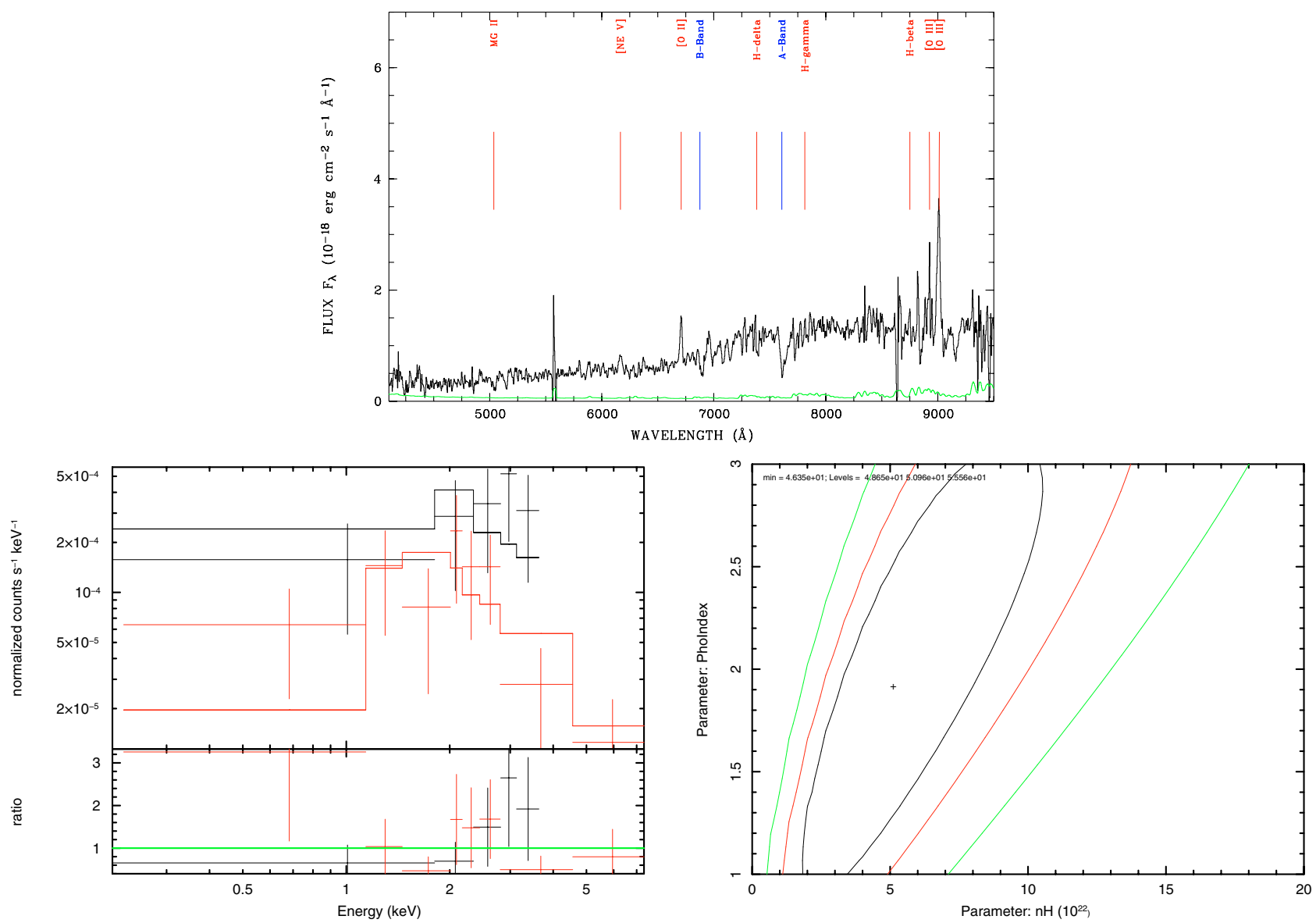

object Marano 171A, $z=0.800$, frozen $\Gamma=2.0$ X-ray spectrum fit.
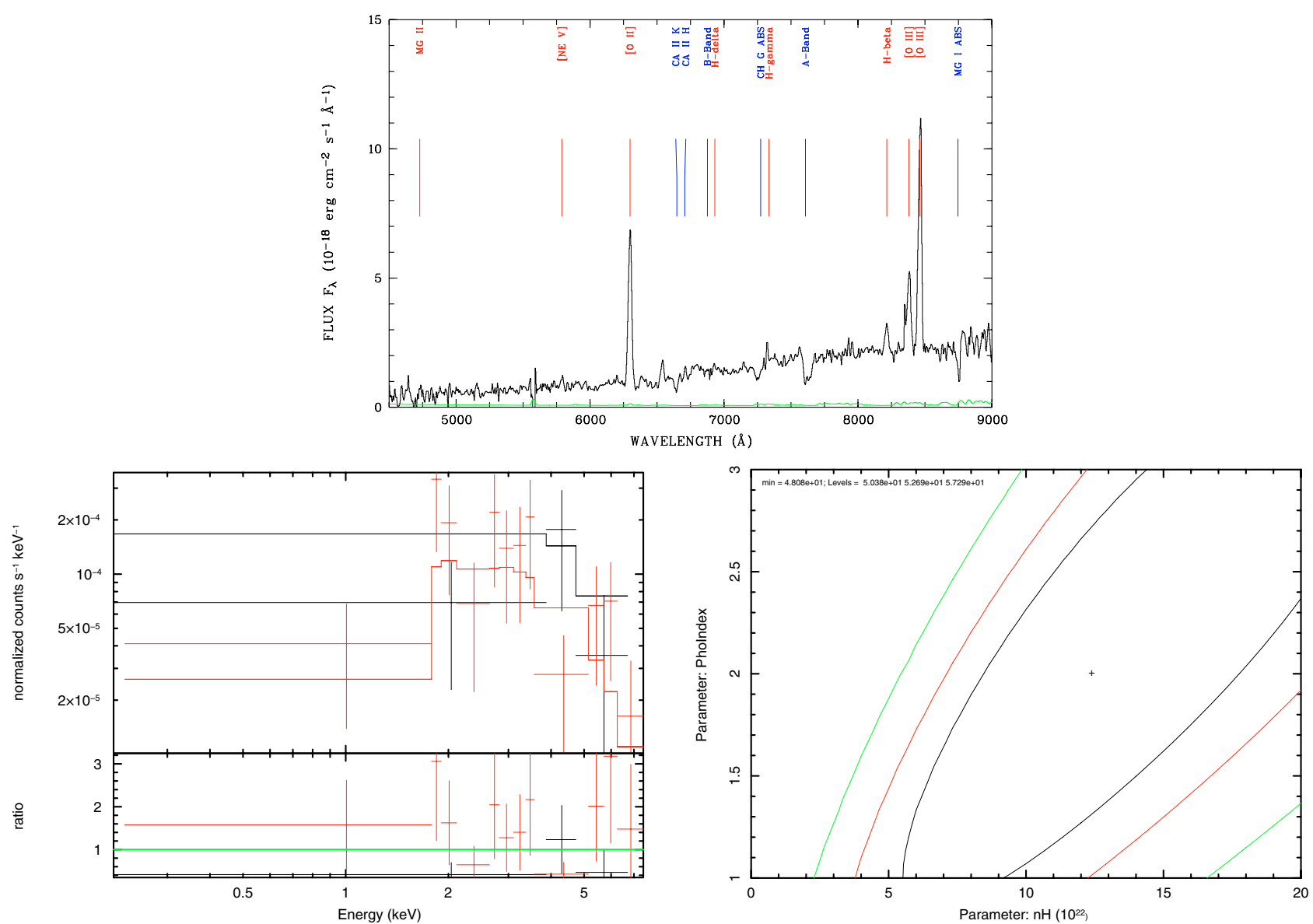

object Marano 224B, $z=0.690$, frozen $\Gamma=2.0$ X-ray spectrum fit. 
M. Krumpe et al.: X-ray absorption in distant type II QSOs, Online Material $p 8$
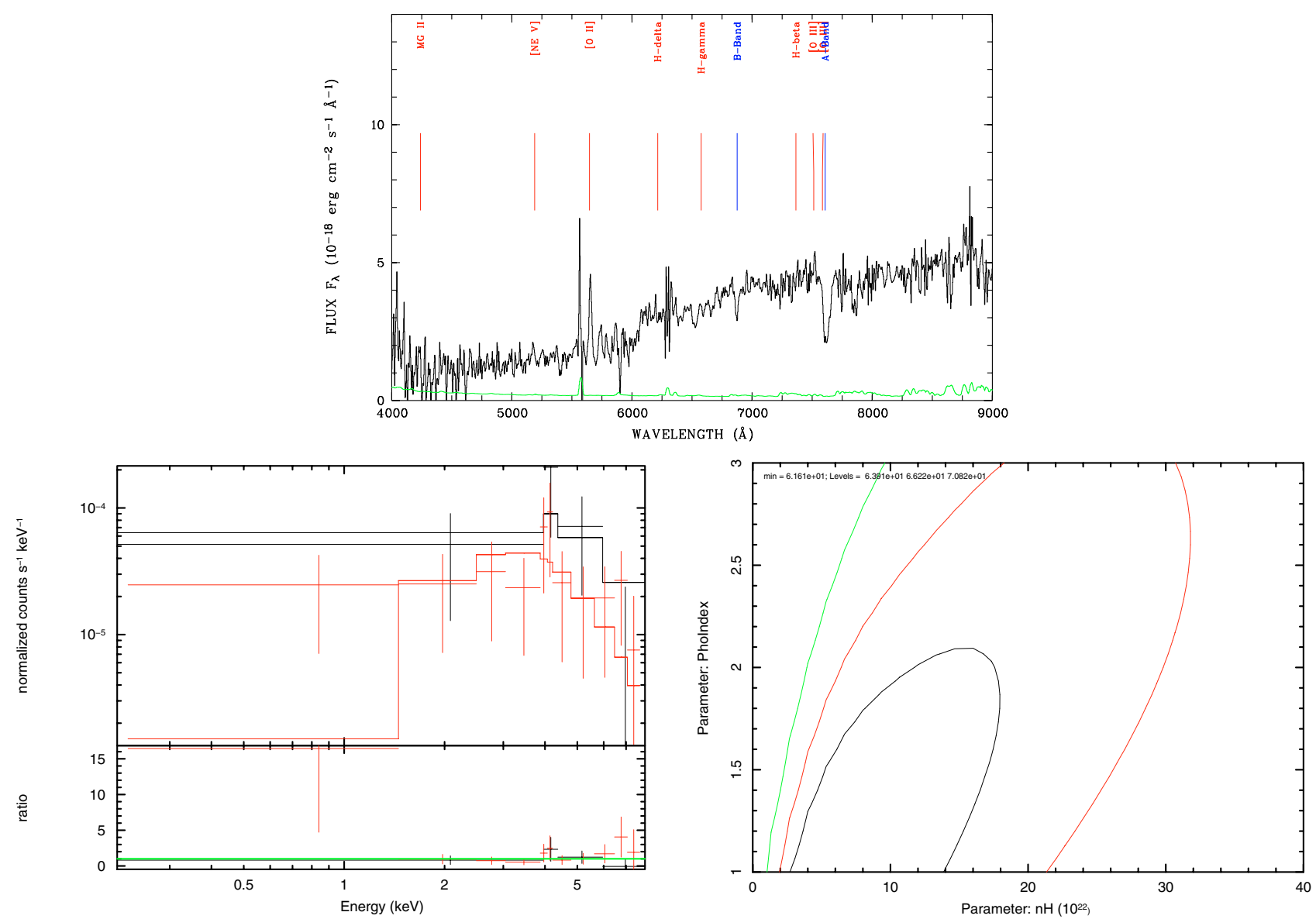

object Marano 253A, $z=0.517$, frozen $\Gamma_{+}=2.0$ X-ray spectrum fit.
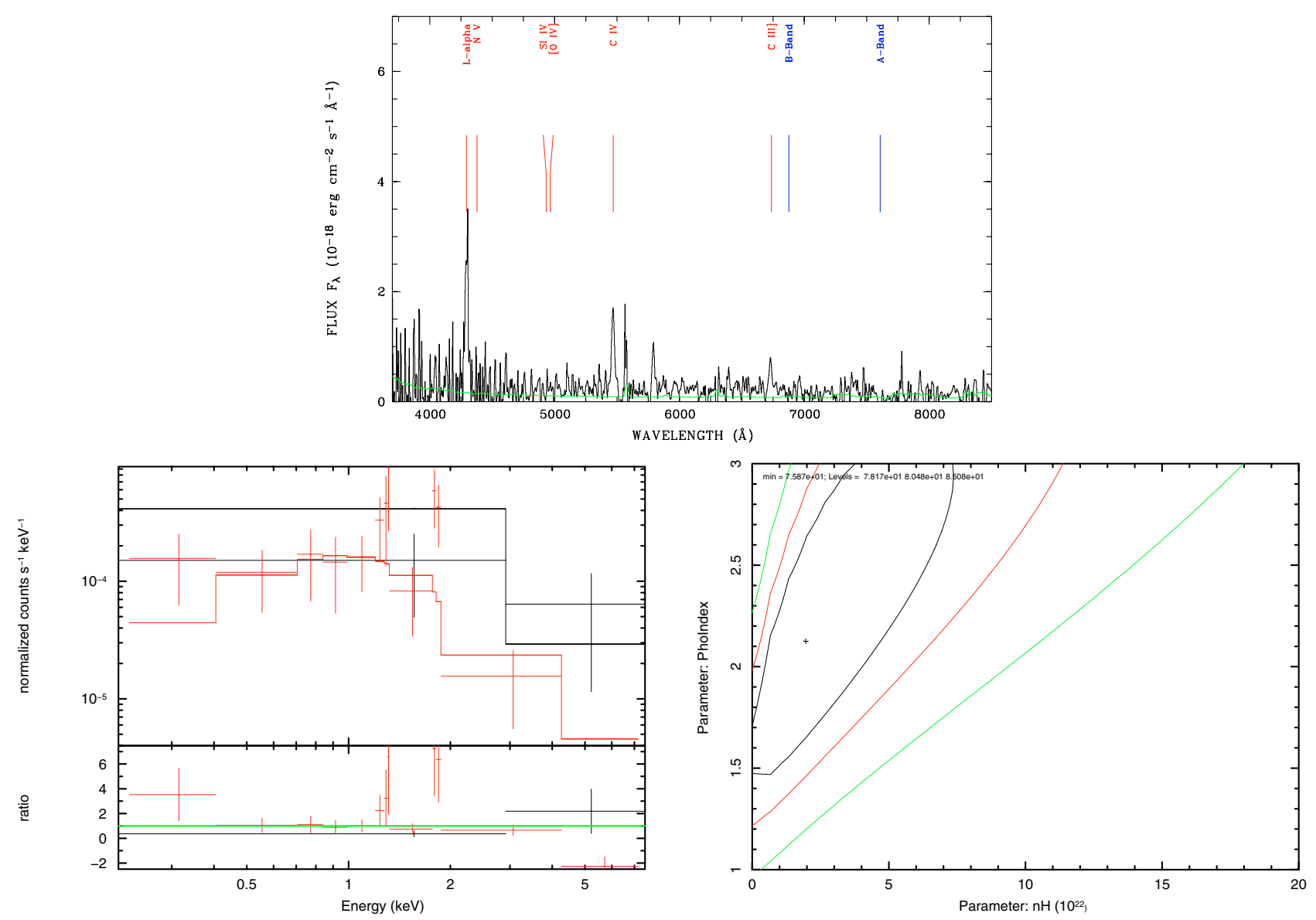

object Marano 463A, $z=2.531$, frozen $\Gamma=2.0$ X-ray spectrum fit. 
M. Krumpe et al.: X-ray absorption in distant type II QSOs, Online Material $p 9$
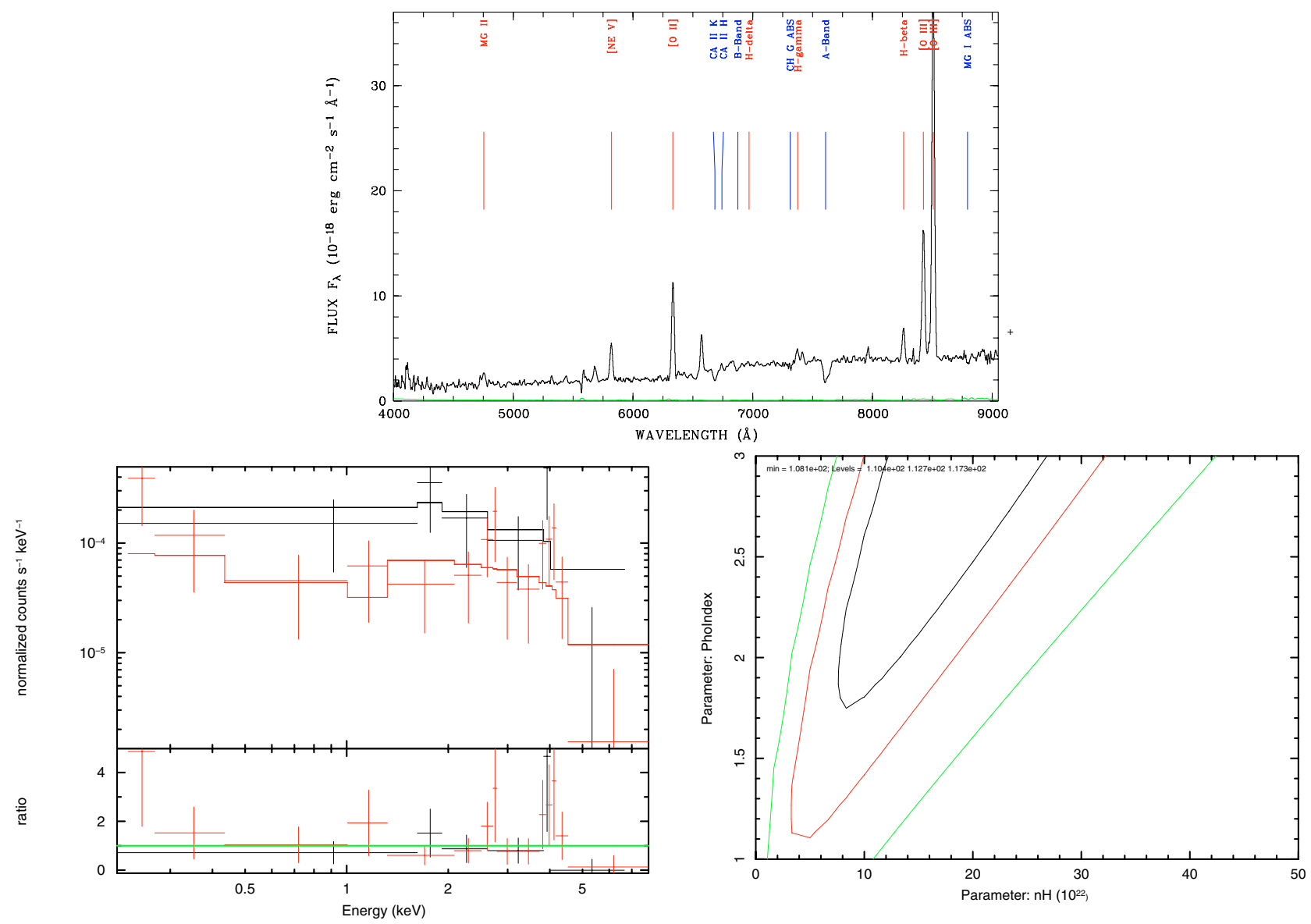

object Marano 610A, $z=0.699$, two power laws, frozen $\Gamma=2.0$ X-ray spectrum fit.
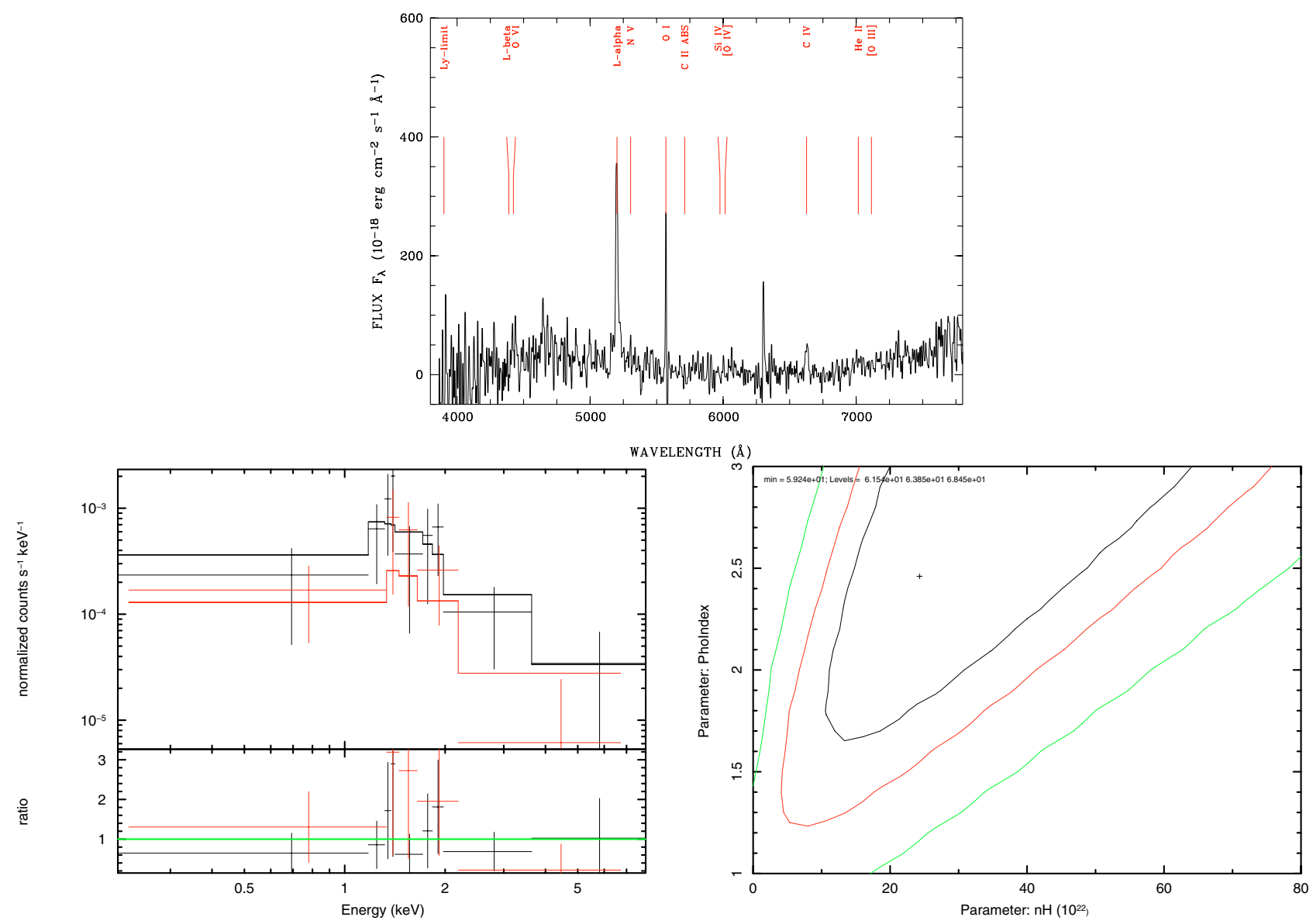

object X21516_135, $z=3.278$, frozen $\Gamma=2.0$ X-ray spectrum fit. 
M. Krumpe et al.: X-ray absorption in distant type II QSOs, Online Material $p 10$
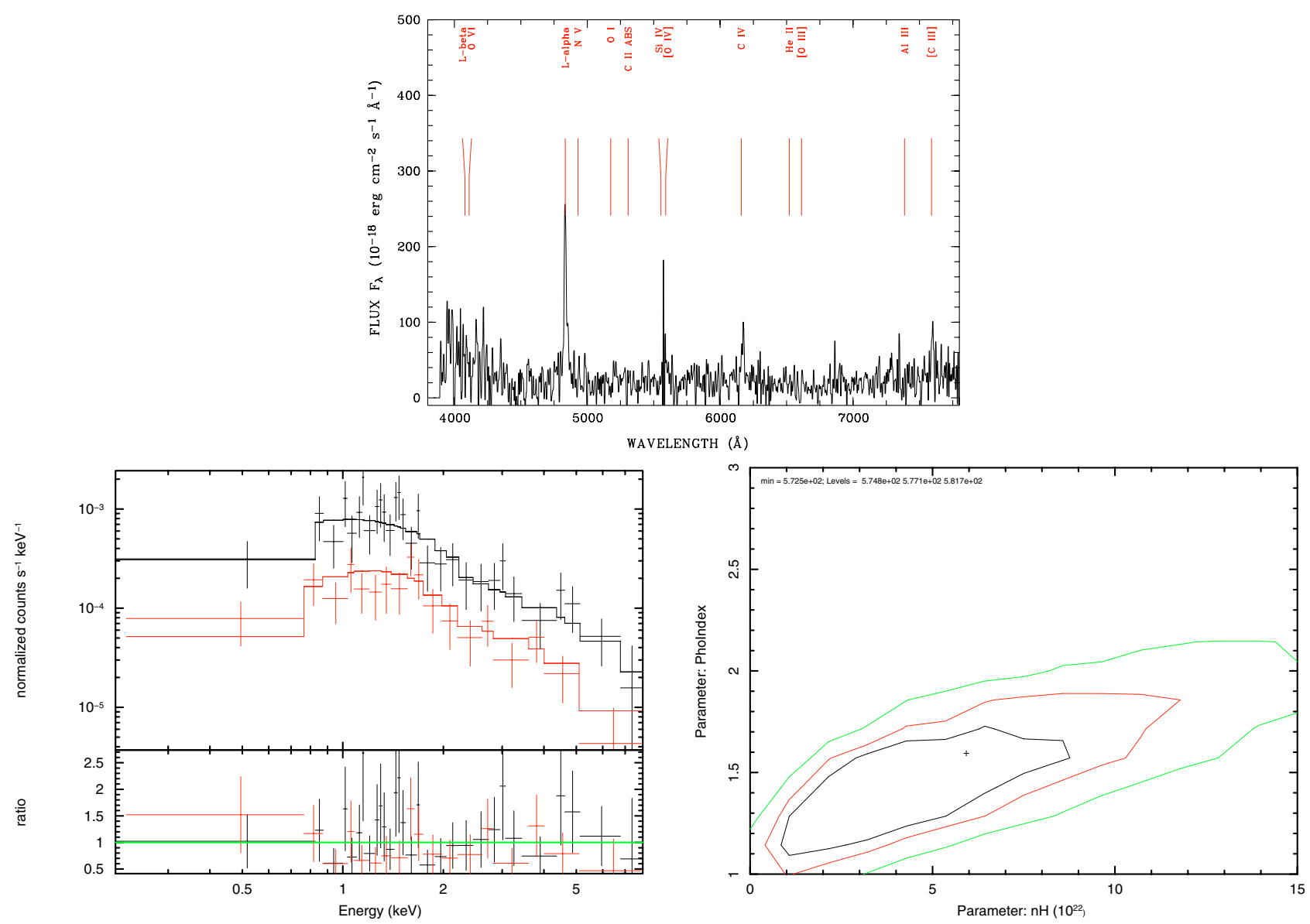

object X00851_154, $z=2.976$, free X-ray spectrum fit.
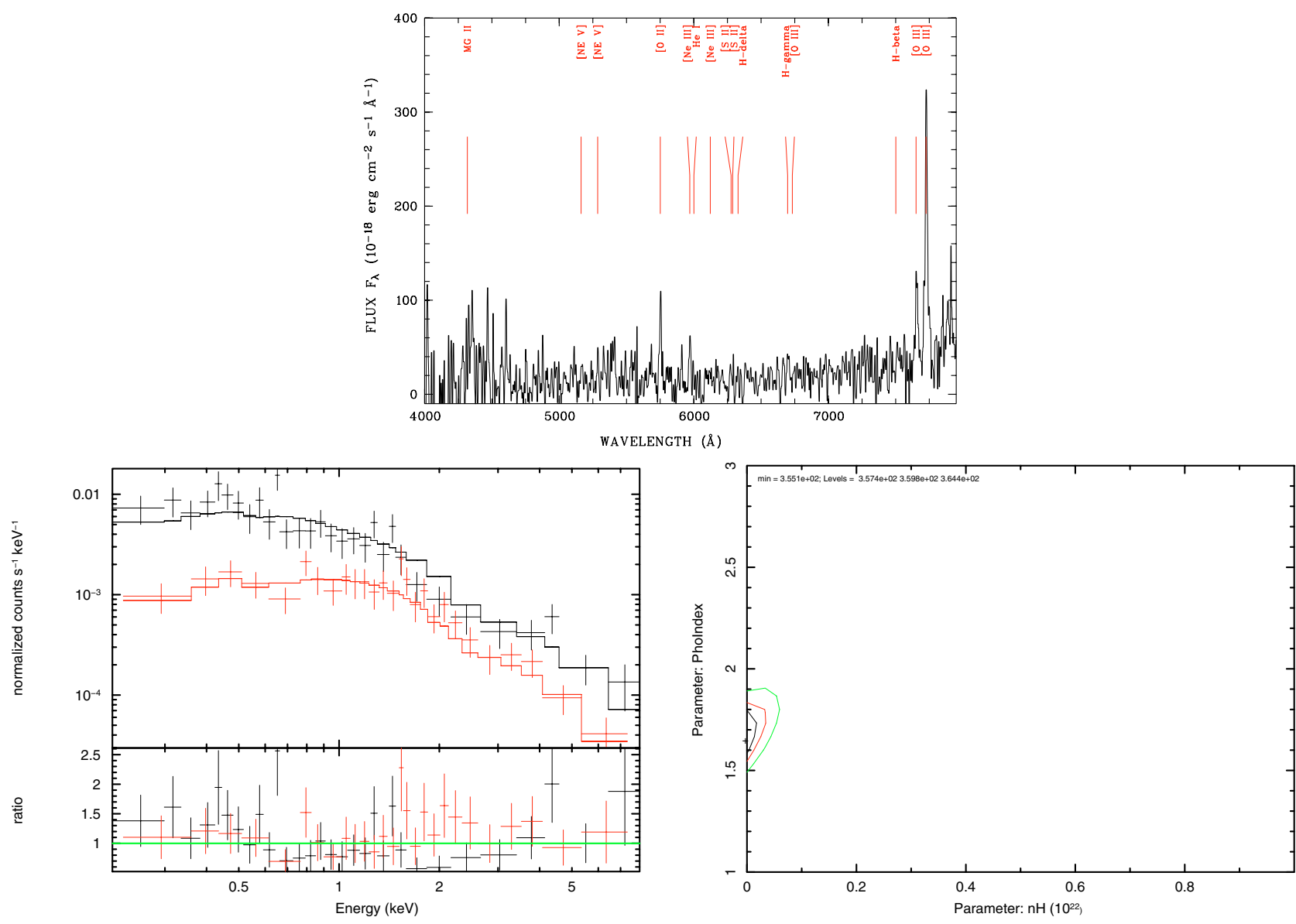

object X01135_126, $z=0.543$, free X-ray spectrum fit. 
M. Krumpe et al.: X-ray absorption in distant type II QSOs, Online Material p 11
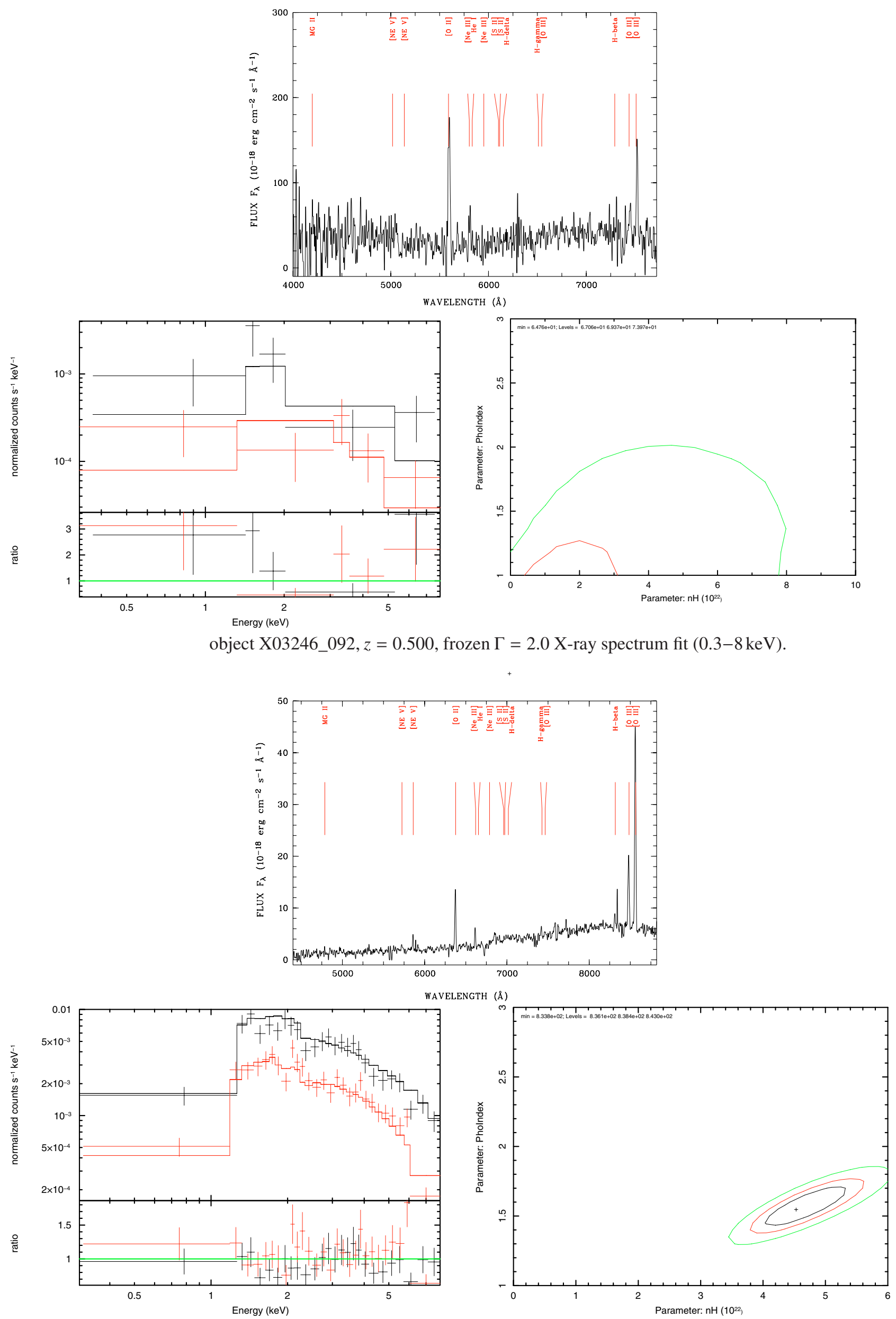

object phl5200-001, $z=0.711$, free X-ray spectrum fit. 
M. Krumpe et al.: X-ray absorption in distant type II QSOs, Online Material p 12
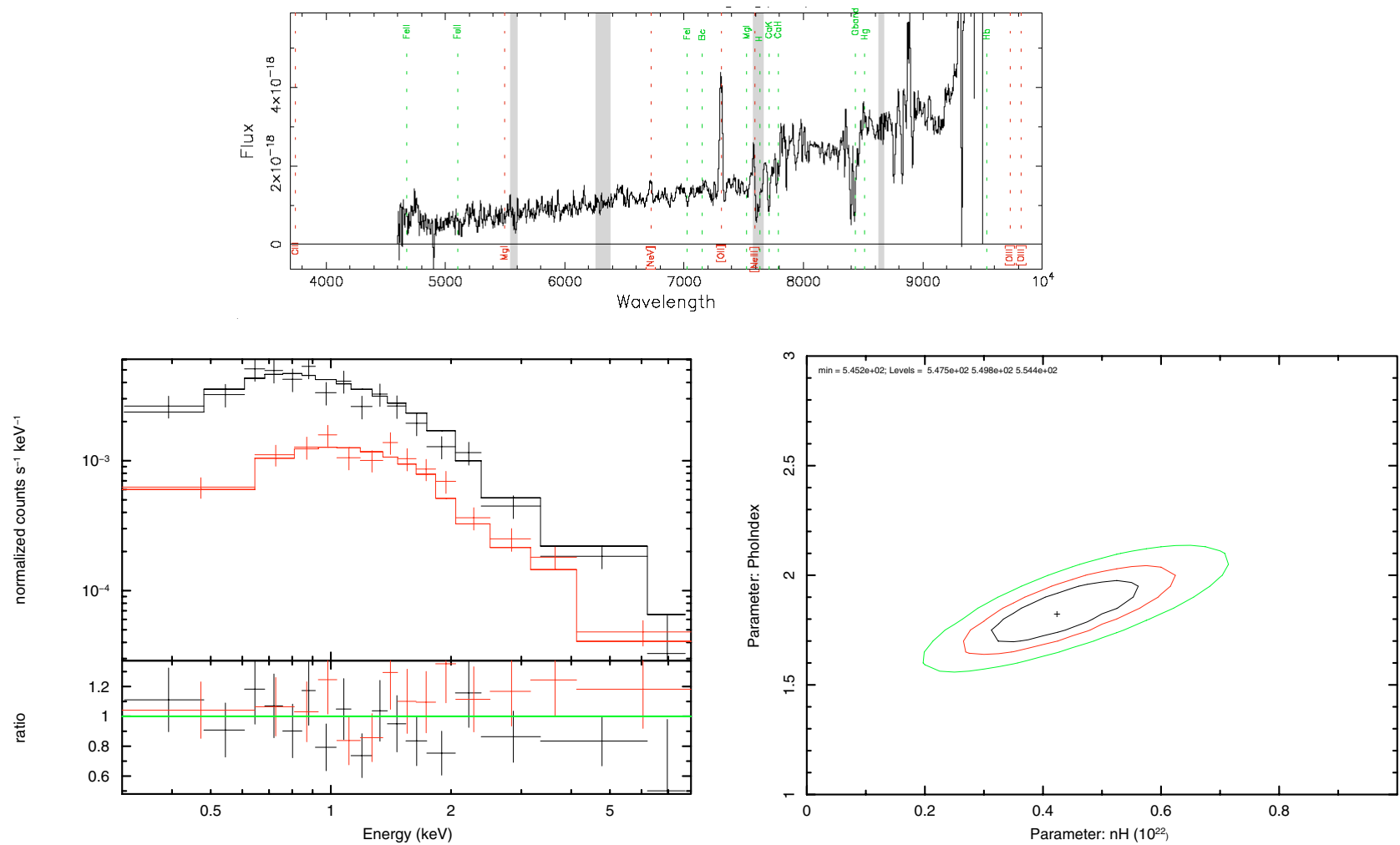

object sds1b-014, $z=0.962$, free X-ray spectrum fit.

(optical spectrum taken from Akiyama et al. 2008). 